\title{
Tree-level S-matrix of superstring field theory with homotopy algebra structure
}

\author{
Hiroshi Kunitomo \\ Center for Gravitational Physics, Yukawa Institute for Theoretical Physics, Kyoto University, \\ Kitashirakawa Oiwakecho, Sakyo-ku, Kyoto 606-8502, Japan \\ E-mail: kunitomo@yukawa.kyoto-u.ac.jp
}

ABStRaCT: We show that the tree-level S-matrices of the superstring field theories based on the homotopy-algebra structure agree with those obtained in the first-quantized formulation. The proof is given in detail for the heterotic string field theory. The extensions to the type II and open superstring field theories are straightforward.

KEYwords: String Field Theory, Superstrings and Heterotic Strings

ARXIV EPRINT: 2011.11975 


\section{Contents}

1 Introduction 1

2 Tree-level S-matrix of heterotic string field theory 3

2.1 Heterotic string field theory with cyclic $L_{\infty}$ structure 3

2.2 S-matrix generating function 8

$\begin{array}{lll}2.3 & \text { Evaluation of the S-matrix } & 11\end{array}$

$\begin{array}{lll}3 & \text { Extension to the type II superstring field theory } & \mathbf{1 5}\end{array}$

$\begin{array}{lll}3.1 & \text { Type II superstring field theory with } L_{\infty} \text { structure } & 15\end{array}$

$\begin{array}{lll}3.2 & \text { S-matrix generating function and its evaluation } & 18\end{array}$

4 Extension to the open superstring field theory $\quad 19$

4.1 Open superstring field theory with cyclic $A_{\infty}$ structure 20

$\begin{array}{lll}4.2 & \text { S-matrix generating function and its evaluation } & 22\end{array}$

5 Summary and discussion $\quad 23$

$\begin{array}{ll}\text { A S-matrix via HPT } & 24\end{array}$

$\begin{array}{ll}\text { B Derivation of }(2.54 a) & 31\end{array}$

C Proof of (2.69) 32

$\begin{array}{ll}\text { D Proof of (2.70) } & 33\end{array}$

E Relation to the Erler-Okawa-Takezaki open superstring field theory $\quad 34$

$\begin{array}{ll}\text { F Cyclicity of generalized } A_{\infty} \text { structure } & 36\end{array}$

\section{Introduction}

After the pioneering work by Witten [1], research on the superstring field theory was falling into a long period of stagnation, except for a few important developments [2-6]. Recently, however, several important progress has been made one after another [7-25], and several complete superstring field theories are now established.

Now, there are three complementary formulations, each of which has advantages and disadvantages: the Wess-Zumino-Witten (WZW) -like formulation, the formulation based on the homotopy-algebra structure, and the formulation accompanied with an extra free field. The WZW-like formulation was first proposed for the open superstring by Berkovits in his ingenious paper [4] and afterward extended to the heterotic string field theory $[5,6]$. 
Although both of them were originally limited to the NS sector, but have recently been extended to a complete form including the Ramond sector [16, 24]. Attempts to construct a WZW-like action for the type II superstring field theory are also being made [22, 25]. The homotopy-algebra-based formulation, the open superstring field theory with an $A_{\infty}$ structure [9], and the heterotic and type II superstring field theories with an $L_{\infty}$ structure [10], was pioneered by the Munich group. Also in this formulation, all the constructions were initially limited to the NS or NS-NS sector, but soon have been extended to those including the Ramond sector, and now completed [17, 24, 25]. The formulation accompanied with an extra free field has been developed by Sen for the heterotic and type II superstring field theories $[12,15]$. In this formulation, a pair of Ramond string fields are introduced, which double the degrees of freedom but half of them is cleverly decoupled from the physical world as a free field. It has been shown that the formulation can also apply to the open superstring field theory [18].

In this paper, we consider the homotopy-algebra-based superstring field theories and show that their tree-level physical S-matrices agree with those calculated by the firstquantized method [26, 27]. In the bosonic string theory, the amplitude for each process is given by the integration over the moduli space of punctured Riemann surface. The string field theory provides a triangulation of the moduli space, each region of which is filled with the contribution from a Feynman diagram. Each contribution is necessary to be connected smoothly at the boundaries so that the sum is an integral over the entire moduli space. It is well known that this requirement is essentially equivalent to requiring the action to be gauge invariant [28-31]. For the superstring field theories, on the other hand, the amplitudes are given by the integral over the super moduli space, so we must also take into account the contribution of the odd moduli integration. It can be incorporated by insertions of the picture changing operator (PCO) [27], which apparently seems to disturb the smooth connection between contributions from Feynman diagrams. However, the gauge-invariant action constructed by utilizing the homotopy algebra structure also includes terms that may fill the gap as contributions from the vertical integration [32]. As the result, the superstring field theories with homotopy algebra structure reproduce the S-matrices obtained in the first-quantized formulation, which the purpose of this paper is to prove.

The study along this direction was previously performed for several tree-level fourstring amplitudes: four-NS string amplitude in the open superstring [9], and arbitrary four-string amplitudes in the heterotic string field theory [24], and some typical four-string amplitudes in the type II superstring field theory [25]. ${ }^{1}$ In addition to these confirmations by explicit calculation, a general proof for the tree-level S-matrix was also given based on the minimal model with the homological perturbation theory (HPT) [35]. This proof is, however, still restricted to the amplitudes with the external NS(-NS) strings, and the proof for the complete S-matrix is still lacking. We extend to the proof for the general S-matrix and complete the proof in the homotopy-algebra-based superstring field theories.

\footnotetext{
${ }^{1}$ The explicit confirmations for four-NS [33] and arbitrary four- and five-string amplitudes [34] were also performed in the WZW-like formulation. These can also be considered as a confirmation of those in the homotopy-algebra-based formulation since the two theories are related by a simple field redefinition.
} 
The paper is organized as follows. In section 2, we give a general proof for the heterotic string in detail. We first summarize some basic properties of the heterotic string field theory with cyclic $L_{\infty}$ structure in section 2.1, focusing on what is needed for the proof. Then, in section 2.2, we give the S-matrix generating function at tree-level in a closed form based on a general argument given in refs. [36, 37]. The result agrees with that given by using the argument based on the (almost) minimal model with HPT. Using the S-matrix generating function, we show that the tree-level physical S-matrix agrees with that calculated using the first-quantized method in section 2.3. The proof is given by generalizing the method used in ref. [35]. It is straightforward to extend the proof to the type II superstring field theory, which is given in section 3. After summarizing the basic properties in section 3.1, we give a proof for type II superstring field theory in section 3.2. The extension to the open superstring field theory is also straightforward if we generalize the construction method in ref. [17] to the one applicable more general $A_{\infty}$ structure following the way given in ref. [24]. Starting from giving such a generalized open superstring field theory in section 4.1 , we prove the agreement of the S-matrix in section 4.2. Section 5 is devoted to the summary and discussion. Finally, six appendices are included. Appendix B is devoted to deriving the final form of the S-matrix generating functional, which is not given in the text since some technical details are required. In appendix A, we explain how the S-matrix is also obtained as the (almost) minimal model via HPT. Some proofs to confirm the consistency of the perturbation are also given. Appendices $\mathrm{C}$ and $\mathrm{D}$ are devoted to proving some formulas used in the text. In appendix E, we show that the generalized open superstring field theory given in section 4 reduces to that in ref. [17] as a special case based on the Witten's vertex. A simple proof for the cyclicity of the $A_{\infty}$ structure in the generalized theory is also given in appendix F, which was not so simple in the previous construction.

\section{Tree-level S-matrix of heterotic string field theory}

Let us first take the heterotic string field theory and show that it reproduces the tree-level S-matrix calculated by the first-quantized method.

\subsection{Heterotic string field theory with cyclic $L_{\infty}$ structure}

We start by briefly summarizing how the heterotic string field theory based on the $L_{\infty}$ structure is constructed. The first-quantized heterotic string theory is obtained by combining the left-moving (holomorphic) superconformal field theory and the right-moving (anti-holomorphic) bosonic conformal field theory. The former consists of a matter sector with $c=15$, the fermionic (conformal) ghosts $(b, c)$, and the bosonic (superconformal) ghosts $(\beta, \gamma)$. The latter consists of a matter sector with $c=26$, and the fermionic ghosts $(\bar{b}, \bar{c})$. It is useful to 'bosonize' the bosonic ghosts $(\beta, \gamma)$ to a pair of fermions $(\eta, \xi)$ and a chiral boson $\phi$. The Hilbert space of the bosonized ghosts $(\eta, \xi ; \phi)$ is called the large Hilbert space $\mathcal{H}_{l}$. In contrast, the Hilbert space of the bosonic ghosts $(\beta, \gamma)$ is called the small Hilbert space $\mathcal{H}_{s}$, which can be embedded in $\mathcal{H}_{l}$ as states $\Phi$ satisfying $\eta \Phi=0$ with a fixed picture number. ${ }^{2}$ The subspaces with different picture numbers provide equivalent

\footnotetext{
${ }^{2}$ For notational simplicity, the zero-mode of $\eta$-ghost appearing frequently is simply denoted as $\eta$.
} 
representations of $\mathcal{H}_{s}$, and can be related to each other by one-to-one mapping using PCO. The heterotic string field takes a value in $\mathcal{H}_{s}$ as described below in detail.

The heterotic string field $\Phi$ is Grassmann even and satisfying the closed string constraints:

$$
b_{0}^{-} \Phi=L_{0}^{-} \Phi=0 .
$$

It has ghost number 2 and two components

$$
\Phi=\Phi_{\mathrm{NS}}+\Phi_{R} \in \mathcal{H}^{\mathrm{res}}=\mathcal{H}_{\mathrm{NS}}+\mathcal{H}_{R}^{\mathrm{res}}
$$

where $\mathcal{H}_{\mathrm{NS}}\left(\mathcal{H}_{R}\right)$ is the small Hilbert space of the NS (Ramond) sector with picture number $-1(-1 / 2)$. The Ramond Hilbert space $\mathcal{H}_{R}^{\text {res }}$ is further restricted by an extra condition:

$$
\mathcal{H}_{R}^{\text {res }}=\left\{\Phi_{R} \in \mathcal{H}_{R} \mid X Y \Phi_{R}=\Phi_{R}\right\},
$$

where $X$ and $Y$ are defined by

$$
X=-\delta\left(\beta_{0}\right) G_{0}+\delta^{\prime}\left(\beta_{0}\right) b_{0}, \quad Y=-2 c_{0}^{+} \delta^{\prime}\left(\gamma_{0}\right) .
$$

The operator $X$ is the PCO on states with picture number $-3 / 2$, and commutative with the BRST operator $Q$. In the large Hilbert space, it can be written as the BRST exact form $X=\{Q, \Xi\}$ with

$$
\Xi=\xi_{0}+\left(\Theta\left(\beta_{0}\right) \eta \xi_{0}-\xi_{0}\right) \Pi_{-3 / 2}+\left(\xi_{0} \eta \Theta\left(\beta_{0}\right)-\xi_{0}\right) \Pi_{-1 / 2} .
$$

Here, $\Pi_{n}$ is the projection operator onto the states with picture number $n$. The operator $Y$ acts on states with picture number $-1 / 2$ as an inverse of $X$ in the sense that it satisfies

$$
X Y X=X, \quad Y X Y=Y .
$$

We can show that $X Y$ used to define $\mathcal{H}^{\text {res }}$ is a projection operator acting on the sates with picture number $-1 / 2$. The ghost number of the string field is equal to that of the basis states for the classical string field.

For later use, it is useful to introduce a notation

$$
\mathcal{G}=\pi^{0}+X \pi^{1}, \quad \mathcal{G}^{-1}=\pi^{0}+Y \pi^{1},
$$

with the projection operator $\pi^{0}\left(\pi^{1}\right)$ onto the NS (Ramond) component. Then, the restricted Hilbert space $\mathcal{H}^{\text {res }}$ can concisely be written as

$$
\mathcal{H}^{\mathrm{res}}=\left\{\Phi \in \mathcal{H}_{s}=\mathcal{H}_{\mathrm{NS}}+\mathcal{H}_{R} \mid \mathcal{G G}^{-1} \Phi=\Phi\right\} .
$$

The restricted Hilbert space $\mathcal{H}^{\text {res }}$ is closed under the action of the BRST operator: $X Y Q X Y=Q X Y$, and any state $\mathcal{B} \in \mathcal{H}^{\text {res }}$ can be expanded in the ghost zero-mode as

$$
\begin{aligned}
\mathcal{B} & =\mathcal{B}_{\mathrm{NS}}+\mathcal{B}_{R} \\
& =\left(b_{\mathrm{NS}}-c_{0}^{+} B_{\mathrm{NS}}\right)+\left(b_{R}-\frac{1}{2}\left(\gamma_{0}+2 c_{0}^{+} G\right) B_{R}\right),
\end{aligned}
$$


where $G=G_{0}+2 \gamma_{0} b_{0}$. In particular, we denote the string field $\Phi \in \mathcal{H}^{\text {res }}$ as

$$
\Phi=\left(\phi_{\mathrm{NS}}-c_{0}^{+} \psi_{\mathrm{NS}}\right)+\left(\phi_{R}-\frac{1}{2}\left(\gamma_{0}+2 c_{0}^{+} G\right) \psi_{R}\right) .
$$

We can define three symplectic forms for the large, small and restricted Hilbert space, $\omega_{l}, \omega_{s}$ and $\Omega$ by

$$
\begin{aligned}
\omega_{l}\left(\Phi_{1}, \Phi_{2}\right) & =(-1)^{\left|\Phi_{1}\right|}{ }_{l}\left\langle\Phi_{1}\left|c_{0}^{-}\right| \Phi_{2}\right\rangle_{l}, & & \Phi_{1}, \Phi_{2} \in \mathcal{H}_{l}, \\
\omega_{s}\left(\Phi_{1}, \Phi_{2}\right) & =(-1)^{\left|\Phi_{1}\right|}{ }_{s}\left\langle\Phi_{1}\left|c_{0}^{-}\right| \Phi_{2}\right\rangle_{s}, & & \Phi_{1}, \Phi_{2} \in \mathcal{H}_{s} \\
\Omega\left(\Phi_{1}, \Phi_{2}\right) & =(-1)^{\left|\Phi_{1}\right|}{ }_{s}\left\langle\Phi_{1}\left|c_{0}^{-} \mathcal{G}^{-1}\right| \Phi_{2}\right\rangle_{s}, & & \Phi_{1}, \Phi_{2} \in \mathcal{H}^{\mathrm{res}}
\end{aligned}
$$

For $\Phi_{1}, \Phi_{2} \in \mathcal{H}^{\text {res }}$, they are related as

$$
\Omega\left(\Phi_{1}, \Phi_{2}\right)=\omega_{s}\left(\Phi_{1}, \mathcal{G}^{-1} \Phi_{2}\right)=\omega_{l}\left(\xi_{0} \Phi_{1}, \mathcal{G}^{-1} \Phi_{2}\right) .
$$

We also use their bilinear map representation defined by

$$
\begin{array}{ccc}
\left\langle\omega_{l}\right|: \mathcal{H}_{l} \otimes \mathcal{H}_{l} & \longrightarrow & \mathbb{C} \\
\Psi & & \Psi \\
\Phi_{1} \otimes \Phi_{2} & \longmapsto & \omega_{l}\left(\Phi_{1}, \Phi_{2}\right),
\end{array}
$$

and

$$
\left\langle\omega_{s}\right|=\left\langle\omega_{l}\right|\left(\xi_{0} \otimes \mathbb{I}\right), \quad\langle\Omega|=\left\langle\omega_{l}\right|\left(\xi_{0} \otimes \mathcal{G}^{-1}\right) .
$$

Note that the natural inner product in $\mathcal{H}^{\text {res }}$ defined by $\Omega$ has the off-diagonal form

$$
\Omega\left(\Phi_{1}, \Phi_{2}\right)=\left\langle\left\langle\phi_{N S 1} \mid \psi_{N S 2}\right\rangle\right\rangle+\left\langle\left\langle\psi_{N S 1} \mid \phi_{N S 2}\right\rangle\right\rangle+\left\langle\left\langle\phi_{R 1} \mid \psi_{R 2}\right\rangle\right\rangle+\left\langle\left\langle\psi_{R 1} \mid \phi_{R 2}\right\rangle\right\rangle,
$$

after integrating out the ghost zero-modes. ${ }^{3}$

The action of heterotic string field theory with cyclic $L_{\infty}$ structure is written as

$$
I[\Phi]=\sum_{n=0}^{\infty} \frac{1}{(n+2) !} \Omega\left(\Phi, L_{n+1}(\underbrace{\Phi, \cdots, \Phi}_{n+1})\right),
$$

which is invariant under the gauge transformation

$$
\delta \Phi=\sum_{n=0}^{\infty} \frac{1}{n !} L_{n+1}(\underbrace{\Phi, \cdots, \Phi}_{n}, \Lambda) .
$$

Here, $L_{1}=Q$ and the multi-string products $L_{n+2}\left(\Phi_{1}, \cdots, \Phi_{n+2}\right)$ are graded commutative, which satisfy the $L_{\infty}$ relations

$$
\sum_{\sigma} \sum_{m=1}^{n}(-1)^{\epsilon(\sigma)} \frac{1}{m !(n-m) !} L_{n-m+1}\left(L_{m}\left(\Phi_{\sigma(1)}, \cdots, \Phi_{\sigma(m)}\right), \Phi_{\sigma(m+1)}, \cdots, \Phi_{\sigma(n)}\right)=0
$$

\footnotetext{
${ }^{3}$ The double bra-ket represents the BPZ inner-product after integrating out the ghost zero-modes [25].
} 
and the cyclicity condition

$$
\Omega\left(\Phi_{1}, L_{n}\left(\Phi_{2}, \cdots, \Phi_{n+1}\right)\right)=-(-1)^{\left|\Phi_{1}\right|} \Omega\left(L_{n}\left(\Phi_{1}, \cdots, \Phi_{n}\right), \Phi_{n+1}\right) .
$$

The symbol $\sigma$ in (2.18) denotes the permutation from $\{1, \cdots, n\}$ to $\{\sigma(1), \cdots, \sigma(n)\}$ and the factor $\epsilon(\sigma)$ is the sign factor of permutation of string fields from $\left\{\Phi_{1}, \cdots, \Phi_{n}\right\}$ to $\left\{\Phi_{\sigma(1)}, \cdots, \Phi_{\sigma(n)}\right\}$. The set of the string products satisfying these conditions is called a cyclic $L_{\infty}$ algebra $\left(\mathcal{H}^{\text {res }}, \Omega,\left\{L_{n}\right\}\right)$.

For constructing the string products of the cyclic $L_{\infty}$ algebra, we use the coalgebra representation, which is, for example, summarized in [24, 38]. The heterotic string products are represented by the (Grassmann) odd coderivation $\boldsymbol{L}=\boldsymbol{Q}+\boldsymbol{L}_{\text {int }}$ acting on the symmetrized tensor algebra $\mathcal{S} \mathcal{H}^{\text {res }}=\oplus_{n=0}^{\infty}\left(\mathcal{H}^{\text {res }}\right)^{\wedge n}$. However, it is not very good idea to directly construct the cyclic $L_{\infty}$ algebra $\left(\mathcal{H}^{\text {res }}, \Omega, \boldsymbol{L}\right)$ since $\Omega$ is asymmetric between the NS and Ramond sectors. Instead, we considered, in [24], a cyclic $L_{\infty}$ algebra $\left(\mathcal{H}_{l}, \omega_{l}, \boldsymbol{Q}-\boldsymbol{\eta}+\boldsymbol{B}\right)$ first. The coderivation $\boldsymbol{B}$ acting on $\mathcal{S H}_{l}=\oplus_{n=0}^{\infty}\left(\mathcal{H}_{l}\right)^{\wedge n}$ is constructed so that an extended generating function,

$$
\boldsymbol{B}(s, t)=\left.\sum_{m, n, r=0}^{\infty} s^{m} t^{n} \boldsymbol{B}_{m+n+r+1}^{(n)}\right|^{2 r}
$$

related to $\boldsymbol{B}$ as $\boldsymbol{B}=\boldsymbol{B}(0,1)$, satisfies two differential equations

$$
\begin{aligned}
\partial_{t} \boldsymbol{B}(s, t) & =[\boldsymbol{Q}, \boldsymbol{\lambda}(s, t)]+[\boldsymbol{B}(s, t), \boldsymbol{\lambda}(s, t)]^{1}+s[\boldsymbol{B}(s, t), \boldsymbol{\lambda}(s, t)]^{2}, \\
\partial_{s} \boldsymbol{B}(s, t) & =[\boldsymbol{\eta}, \boldsymbol{\lambda}(s, t)]-t[\boldsymbol{B}(s, t), \boldsymbol{\lambda}(s, t)]^{2},
\end{aligned}
$$

which simultaneously determine another even coderivation

$$
\boldsymbol{\lambda}(s, t)=\left.\sum_{m, n, r=0}^{\infty} s^{m} t^{n} \boldsymbol{\lambda}_{m+n+r+2}^{(n+1)}\right|^{2 r},
$$

called the (generating function of) gauge products. Here, the brackets $[\cdot, \cdot]^{1}$ and $[\cdot, \cdot]^{2}$ are defined from the (graded) commutators by projecting the cyclic Ramond number as

$$
\left[\left.\boldsymbol{A}\right|^{2 r},\left.\boldsymbol{B}\right|^{2 s}\right]^{1}=\left.\left[\left.\boldsymbol{A}\right|^{2 r},\left.\boldsymbol{B}\right|^{2 s}\right]\right|^{2(r+s)}, \quad\left[\left.\boldsymbol{A}\right|^{2 r},\left.\boldsymbol{B}\right|^{2 s}\right]^{2}=\left.\left[\left.\boldsymbol{A}\right|^{2 r},\left.\boldsymbol{B}\right|^{2 s}\right]\right|^{2(r+s-1)} .
$$

For the coderivation $\boldsymbol{B}(s, t)$ constructed to satisfy (2.21), the relations

$$
\begin{aligned}
{[\boldsymbol{Q}, \boldsymbol{B}(s, t)]+\frac{1}{2}[\boldsymbol{B}(s, t), \boldsymbol{B}(s, t)]^{1}+\frac{s}{2}[\boldsymbol{B}(s, t), \boldsymbol{B}(s, t)]^{2} } & =0 \\
{[\boldsymbol{\eta}, \boldsymbol{B}(s, t)]-\frac{t}{2}[\boldsymbol{B}(s, t), \boldsymbol{B}(s, t)]^{2} } & =0
\end{aligned}
$$

hold, which reduce at $(s, t)=(0,1)$ to the $L_{\infty}$ relation for the coderivation $\boldsymbol{Q}-\boldsymbol{\eta}+\boldsymbol{B}$. We have shown that the coderivation $\boldsymbol{L}$ representing the heterotic string products can be obtained from $\boldsymbol{B}$ as

$$
\begin{aligned}
\boldsymbol{L} & =\hat{\boldsymbol{F}}^{-1}\left(\boldsymbol{Q}+\pi^{0} \boldsymbol{B}\right) \hat{\boldsymbol{F}}, \\
\pi_{1} \boldsymbol{L}_{\mathrm{int}} & =\mathcal{G} \pi_{1} \boldsymbol{l}, \quad \pi_{1} \boldsymbol{l}=\pi_{1} \boldsymbol{B} \hat{\boldsymbol{F}} .
\end{aligned}
$$


Here, $\pi_{n}$ is the projection operator

$$
\pi_{n}: \mathcal{S} \mathcal{H}^{\mathrm{res}} \rightarrow\left(\mathcal{H}^{\mathrm{res}}\right)^{\wedge n} .
$$

The invertible cohomomorphism $\hat{\boldsymbol{F}}^{-1}$ is defined by

$$
\pi_{1} \hat{\boldsymbol{F}}^{-1}=\pi_{1} \mathbb{I}-\Xi \pi_{1}^{1} \boldsymbol{B},
$$

with $\pi_{1}^{1}=\pi^{1} \pi_{1}\left(\pi_{1}^{0}=\pi^{0} \pi_{1}\right)$, and $\mathbb{I} \in \mathcal{S} \mathcal{H}^{\text {res }}$ is the (multiplicative) identity of the symmetrized tensor algebra. Using a property of cohomomorphisms

$$
\hat{\boldsymbol{F}}=\pi_{0}+\sum_{n=1}^{\infty} \frac{1}{n !}\left(\pi_{1} \hat{\boldsymbol{F}}\right)^{\wedge n}
$$

and the relation

$$
\pi_{1} \hat{\boldsymbol{F}}=\pi_{1} \mathbb{I}+\Xi \pi_{1}^{1} \boldsymbol{l}
$$

obtained by acting $\hat{\boldsymbol{F}}$ from the right of both sides of (2.27), we can rewrite the second equation in $(2.25 \mathrm{~b})$ as a self-consistent equation, which we will use later:

$$
\pi_{1} \boldsymbol{l}=\sum_{n=0}^{\infty} \pi_{1} \boldsymbol{B}_{n+2}\left(\frac{1}{(n+2) !}\left(\pi_{1} \mathbb{I}+\Xi \pi_{1}^{1} \boldsymbol{l}\right)^{\wedge(n+2)}\right) .
$$

Finally, we note that the bracket $[,]^{1}$ or $[,]^{2}$ can also be defined by projecting the intermediate state onto the NS or Ramond state, respectively. For example, for coderivations $\boldsymbol{A}_{n+1}$ and $\boldsymbol{B}_{m+1}$, we find that

$$
\begin{aligned}
& {\left[\boldsymbol{A}_{n+1}, \boldsymbol{B}_{m+1}\right]^{1}=\boldsymbol{A}_{n+1}\left(\pi_{1}^{0} \boldsymbol{B}_{m+1} \wedge \mathbb{I}_{n}\right)-(-1)^{A B} \boldsymbol{B}_{m+1}\left(\pi_{1}^{0} \boldsymbol{A}_{n+1} \wedge \mathbb{I}_{m}\right),} \\
& {\left[\boldsymbol{A}_{n+1}, \boldsymbol{B}_{m+1}\right]^{2}=\boldsymbol{A}_{n+1}\left(\pi_{1}^{1} \boldsymbol{B}_{m+1} \wedge \mathbb{I}_{n}\right)-(-1)^{A B} \boldsymbol{B}_{m+1}\left(\pi_{1}^{1} \boldsymbol{A}_{n+1} \wedge \mathbb{I}_{m}\right) .}
\end{aligned}
$$

In this definition, it is easy to see that $[]=,[,]^{1}+[,]^{2}$ holds. We can rewrite the relations in eqs. (2.21) and (2.24) using this representation as

$$
\begin{gathered}
\partial_{t} \boldsymbol{B}_{n+2}(s, t)=\left[\boldsymbol{Q}, \boldsymbol{\lambda}_{n+2}(s, t)\right]+\sum_{m=0}^{n-1}\left(\boldsymbol{B}_{m+2}(s, t)\left(\pi(s) \pi_{1} \boldsymbol{\lambda}_{n-m+1}(s, t) \wedge \mathbb{I}_{m+1}\right)\right. \\
\left.-\boldsymbol{\lambda}_{m+2}(s, t)\left(\pi(s) \pi_{1} \boldsymbol{B}_{n-m+1}(s, t) \wedge \mathbb{I}_{m+1}\right)\right), \\
\partial_{s} \boldsymbol{B}_{n+2}(s, t)=\left[\boldsymbol{\eta}, \boldsymbol{\lambda}_{n+2}(s, t)\right]-\sum_{m=0}^{n-1}\left(\begin{array}{c}
\boldsymbol{B}_{m+2}(s, t)\left(t \pi_{1}^{1} \boldsymbol{\lambda}_{n-m+1}(s, t) \wedge \mathbb{I}_{m+1}\right) \\
\left.-\boldsymbol{\lambda}_{m+2}(s, t)\left(t \pi_{1}^{1} \boldsymbol{B}_{n-m+1}(s, t) \wedge \mathbb{I}_{m+1}\right)\right)
\end{array}\right.
\end{gathered}
$$

and

$$
\begin{aligned}
{\left[\boldsymbol{Q}, \boldsymbol{B}_{n+2}(s, t)\right] } & =-\sum_{m=0}^{n-1} \boldsymbol{B}_{m+2}(s, t)\left(\pi(s) \pi_{1} \boldsymbol{B}_{n-m+1}(s, t) \wedge \mathbb{I}_{m+1}\right), \\
{\left[\boldsymbol{\eta}, \boldsymbol{B}_{n+2}(s, t)\right] } & =\sum_{m=0}^{n-1} \boldsymbol{B}_{m+2}(s, t)\left(t \pi_{1}^{1} \boldsymbol{B}_{n-m+1}(s, t) \wedge \mathbb{I}_{m+1}\right),
\end{aligned}
$$

with $\pi_{1} \boldsymbol{B}_{n+2}(s, t)=\pi_{1} \boldsymbol{B}(s, t) \pi_{n+2}, \pi_{1} \boldsymbol{\lambda}_{n+2}(s, t)=\pi_{1} \boldsymbol{\lambda}(s, t) \pi_{n+2}$ and $\pi(s)=\pi^{0}+s \pi^{1}$. We will use these forms of the relations later. 


\section{$2.2 \quad$ S-matrix generating function}

In the quantum field theory, a perturbative amplitude is conventionally calculated utilizing the Feynman's method: drawing the possible (connected and amputated) Feynman diagrams for a specific process, combining the propagators and vertices, and evaluating them with integrating the loop momentum (momenta) if it is the one at the loop level. Also for the open [9], heterotic [24], and type II [25] superstring field theories, we have already calculated various tree-level four-point amplitudes based on the homotopy-algebra-based formulation. ${ }^{4}$ In this section, we discuss all the (tree-level) amplitudes collectively by considering the S-matrix generating functional at the tree level.

We first quantize the theory following the BV formalism. From the off-diagonal form of the inner product $(2.15)$ in $\mathcal{H}^{\text {res }}$, we can identify $\phi=\phi_{\mathrm{NS}}+\phi_{R}$ and $\psi=\psi_{\mathrm{NS}}+\psi_{R}$ in (2.10) to the field and anti-field of the BV formalism in the gauge-fixed basis [41]. In this basis, the classical BV master action has the same form as the classical action (2.16),

$$
I[\Phi]=\sum_{n=0}^{\infty} \frac{1}{(n+2) !} \Omega\left(\Phi, L_{n+1}(\underbrace{\Phi, \cdots, \Phi}_{n+1})\right),
$$

but $\Phi$ is now the quantum string field including the states in $\mathcal{H}^{\text {res }}$ with all the ghost numbers. ${ }^{5}$ The Siegel-Ramond (SR) gauge is obtained by simply setting $\psi=0$. We call the Hilbert space of the quantum field in this gauge $\mathcal{H}_{\mathrm{SR}}: \Phi=\phi \in \mathcal{H}_{\mathrm{SR}}$. Then the gauge fixed action

$$
I[\phi]=\frac{1}{2}\left\langle\phi\left|c_{0}^{-} \mathcal{G}^{-1} Q\right| \phi\right\rangle+\sum_{n=3}^{\infty} \frac{1}{n !}\left\langle\phi\left|c_{0}^{-}\right| l_{n-1}\left(\phi^{n-1}\right)\right\rangle,
$$

is invariant under the BRST transformation ${ }^{6}$

$$
\delta_{\mathrm{BRST}} \phi=\left.\frac{\partial I[\Phi]}{\partial \psi}\right|_{\psi=0} .
$$

From (2.36), we can read off the propagator

$$
\Pi \equiv-\frac{b_{0}^{+} b_{0}^{-} \mathcal{G}}{L_{0}^{+}} \int_{0}^{2 \pi} \frac{d \theta}{2 \pi} e^{-i \theta L_{0}^{-}},
$$

and the $n$-point vertices

$$
c_{0}^{-} l_{n-1},
$$

for the Feynman rules to calculate amplitudes. Here, however, instead of considering each amplitude independently, we consider the generating functional of S-matrix elements which can be obtained by evaluating the effective action at its stationary configuration [36]. At

\footnotetext{
${ }^{4}$ Several four- and five-point amplitudes have also been calculated by the WZW-like open superstring field theory $[33,34]$.

${ }^{5}$ We use the same symbol for the classical and quantum string fields and their Hilbert spaces, but it would not be confused. The ghost number of the quantum field remains two, which is defined by the sum of two kinds of ghost numbers: the ghost number of basis states and the ghost number of coefficient space-time fields (space-time ghost number).

${ }^{6}$ This is a schematic expression. See ref. [31] for precise treatment.
} 
the tree-level, in particular, it reduces to the classical action evaluated at the classical solution and is calculated as follows.

Let us first define the on-shell subspace,

$$
\mathcal{H}_{0}=\left\{\Phi \in \mathcal{H}^{\mathrm{res}} \mid L_{0}^{+} \Phi_{\mathrm{NS}}=G_{0} \Phi_{R}=0\right\}
$$

and the BRST invariant projection operator,

$$
P_{0}: \mathcal{H}^{\mathrm{res}} \longrightarrow \mathcal{H}_{0}, \quad P_{0}^{2}=P_{0}, \quad\left[Q, P_{0}\right]=0 \text {. }
$$

Then, we introduce an operator

$$
Q^{+}=\frac{1}{L_{0}^{+}} b_{0}^{+}\left(1-P_{0}\right)
$$

satisfying

$$
\begin{array}{r}
Q^{+} Q+Q Q^{+}+P_{0}=\mathbb{I}, \\
Q^{+} P_{0}=P_{0} Q^{+}=Q^{+} Q^{+}=0,
\end{array}
$$

which defines a Hodge-Kodaira decomposition of $\mathcal{H}^{\text {res }}$,

$$
\mathcal{H}^{\mathrm{res}}=\mathcal{H}^{p}+\mathcal{H}^{t}+\mathcal{H}^{u},
$$

with

$$
\mathcal{H}^{p}=P_{0} \mathcal{H}^{\mathrm{res}}, \quad \mathcal{H}^{t}=Q Q^{+} \mathcal{H}^{\mathrm{res}}, \quad \mathcal{H}^{u}=Q^{+} Q \mathcal{H}^{\mathrm{res}} .
$$

This $Q^{+}$is called a (contracting) homotopy operator of $Q$, which is compatible with $\Omega$ :

$$
\Omega\left(\mathcal{H}_{0}, \mathcal{H}^{u}\right)=\Omega\left(\mathcal{H}^{u}, \mathcal{H}^{u}\right)=0 .
$$

Under the SR gauge condition $\psi=0, \mathcal{H}^{t}=\emptyset$ and the quantum field $\phi$ is decomposed to the on-shell and off-shell subspaces:

$$
\phi \in \mathcal{H}_{0}+\overline{\mathcal{H}}_{0},
$$

with

$$
\mathcal{H}_{0}=\mathcal{H}^{p} \cap \mathcal{H}_{\mathrm{SR}}=P_{0} \mathcal{H}_{\mathrm{SR}}, \quad \overline{\mathcal{H}}_{0}=\mathcal{H}^{u} \cap \mathcal{H}_{\mathrm{SR}}=\left(1-P_{0}\right) \mathcal{H}_{\mathrm{SR}} .
$$

The classical equation of motion in the SR gauge can now be written in the form of an integral equation:

$$
\phi=\phi_{0}-Q^{+} \pi_{1} \boldsymbol{L}_{\mathrm{int}}\left(e^{\wedge \phi}\right), \quad \phi_{0} \in \mathcal{H}_{0} .
$$

At the tree level, the S-matrix generating functional is given by evaluating the classical action at the solution of the equation (2.48). Its explicit expression can be found using the coalgebra representation as follows. 
Define the homotopy, projection and identity operators, $\boldsymbol{H}, \hat{\boldsymbol{P}}$, and $\hat{\boldsymbol{I}}$ by

$$
\begin{aligned}
\boldsymbol{H} & =\sum_{r, s=0}^{\infty} \frac{1}{(r+s+1) !}\left(-Q^{+}\right) \wedge \underbrace{\mathbb{I}_{1} \wedge \cdots \wedge \mathbb{I}_{1}}_{r} \wedge \underbrace{P_{0} \wedge \cdots \wedge P_{0}}_{s}, \\
\hat{\boldsymbol{P}} & =\sum_{n=0}^{\infty} P_{n}=\sum_{n=0}^{\infty} \frac{1}{n !} \underbrace{P_{0} \wedge \cdots \wedge P_{0}}_{n}, \\
\hat{\boldsymbol{I}} & =\sum_{n=0}^{\infty} \mathbb{I}_{n}=\sum_{n=0}^{\infty} \frac{1}{n !} \underbrace{\mathbb{I}_{1} \wedge \cdots \wedge \mathbb{I}_{1}}_{n},
\end{aligned}
$$

which act on the symmetric tensor algebra $\mathcal{S} \mathcal{H}_{\mathrm{SR}}$ and satisfy the relations

$$
\begin{aligned}
\hat{\boldsymbol{P}} & =\hat{\boldsymbol{I}}+\boldsymbol{H} \boldsymbol{Q}+\boldsymbol{Q H}, \\
\hat{\boldsymbol{P}}^{2} & =\hat{\boldsymbol{P}}, \quad[\boldsymbol{Q}, \hat{\boldsymbol{P}}]=0, \\
\boldsymbol{H} \hat{\boldsymbol{P}} & =\hat{\boldsymbol{P}} \boldsymbol{H}=\boldsymbol{H} \boldsymbol{H}=0 .
\end{aligned}
$$

Using $Q^{+} \phi=0$ and the general property of the coderivation, we can show that eq. (2.48) is equivalent to

$$
\boldsymbol{H} \boldsymbol{L}\left(e^{\wedge \phi}\right)=0 \text {. }
$$

The solution $\phi_{\mathrm{cl}}$ of this equation can be solved for $\phi_{0}$ as $^{7}$

$$
e^{\wedge \phi_{\mathrm{cl}}}=\left(\hat{\boldsymbol{I}}-\boldsymbol{H} \boldsymbol{L}_{\mathrm{int}}\right)^{-1} \hat{\boldsymbol{P}}\left(e^{\wedge \phi_{0}}\right),
$$

and thus

$$
\phi_{\mathrm{cl}}=\pi_{1}\left(\hat{\boldsymbol{I}}-\boldsymbol{H} \boldsymbol{L}_{\mathrm{int}}\right)^{-1} \hat{\boldsymbol{P}}\left(e^{\wedge \phi_{0}}\right) .
$$

The S-matrix generating functional is a functional of $\phi_{0}$ obtained by evaluating the gauge fixed action (2.36) at the classical solution (2.53), and is given by

$$
\begin{aligned}
S\left[\phi_{0}\right] & \equiv I\left[\phi_{\mathrm{cl}}\right] \\
& =\sum_{n=0}^{\infty} \frac{1}{(n+2) !} \Omega\left(\phi_{0}, S_{n+1}\left(\phi_{0}, \cdots, \phi_{0}\right)\right),
\end{aligned}
$$

with

$$
\boldsymbol{S}=\boldsymbol{Q} \hat{\boldsymbol{P}}+\boldsymbol{S}_{\mathrm{int}}, \quad \boldsymbol{S}_{\mathrm{int}}=\hat{\boldsymbol{P}} \boldsymbol{L}_{\mathrm{int}}\left(\hat{\boldsymbol{I}}-\boldsymbol{H} \boldsymbol{L}_{\mathrm{int}}\right)^{-1} \hat{\boldsymbol{P}},
$$

as is derived in appendix B. This can also be represented by using the multi-linear map ${ }^{8}$ $\langle S|=\sum_{n=0}^{\infty}\left\langle S_{(n+3)}\right|$ with

$$
\begin{array}{rlrl}
\langle S| & =\langle\Omega| P_{0} \otimes \pi_{1} \boldsymbol{S}_{\mathrm{int}}: & \mathcal{H}_{0} \otimes \mathcal{S} \mathcal{H}_{0} & \longrightarrow \mathbb{C}, \\
\left\langle S_{n+3}\right| & =\langle\Omega| P_{0} \otimes \pi_{1} \boldsymbol{S}_{\mathrm{int}} \pi_{n+2}: & \mathcal{H}_{0} \otimes\left(\mathcal{H}_{0}\right)^{\wedge(n+2)} \rightarrow \mathbb{C} .
\end{array}
$$

\footnotetext{
${ }^{7}$ The relation (2.52) shows that the map $\boldsymbol{i}^{\prime} \equiv\left(\hat{\boldsymbol{I}}-\boldsymbol{H} \boldsymbol{L}_{\mathrm{int}}\right)^{-1} \hat{\boldsymbol{P}}$ (deformed inclusion map in the language of HPT) is a cohomomorphism. This was first shown in ref. [39] for the first couple of orders in $\boldsymbol{L}_{\text {int }}$, and subsequently proven in ref. [40]. We also provide an independent proof in appendix A.

${ }^{8}$ Note that the quadratic term in $S\left[\phi_{0}\right]$ vanishes due to the on-shell property of $\phi_{0}$.
} 
The amplitude for $(n+3)$-string scattering can be obtained as

$$
S_{n+3}\left(\phi_{1}, \cdots, \phi_{n+3}\right)=\left\langle S_{n+3}\right|\left(\phi_{1} \otimes \phi_{2} \wedge \cdots \wedge \phi_{n+3}\right),
$$

where $\phi_{i} \in \mathcal{H}_{0}$ are the wave functions (the vertex operators in the first-quantized formulation) of external string states. We can discuss various $(n+3)$-string scattering amplitudes together using $\left\langle S_{n+3}\right|$ (or all the scattering amplitudes using $\langle S|$ ). As an example, the various four-string scattering amplitudes are represented by

$$
\left\langle S_{4}\right|=\langle\Omega| P_{0} \otimes P_{0} \pi_{1}\left(\boldsymbol{L}_{3}-\boldsymbol{L}_{2}\left(\mathbb{I} \wedge Q^{+} \pi_{1} \boldsymbol{L}_{2}\right)\right) P_{3} \pi_{3} .
$$

Taking into account the fact that the output of the product $\pi_{1} \boldsymbol{L}$ satisfies the closed string constraints in eq. (2.1) and has off-shell momentum for inputs with generic momenta, we can rearrange it as

$$
\left\langle S_{4}\right|=\left\langle\omega_{s}\right| P_{0} \otimes P_{0} \pi_{1}\left(\boldsymbol{l}_{3}+\boldsymbol{l}_{2}\left(\mathbb{I} \wedge\left(\frac{b_{0}^{+} \mathcal{G}}{L_{0}^{+}}\right) \pi_{1} \boldsymbol{l}_{2}\right)\right) P_{3} \pi_{3},
$$

from which we can see that the specific amplitude for each process agrees with that calculated by using the Feynman rules in ref. [24].

The S-matrix considered here is the total S-matrix in the covariant BRST formalism. The physical S-matrix is obtained by projecting it (or equivalently the external states) onto the physical subspace $\mathcal{H}_{Q} \subset \mathcal{H}_{0}$ defined by the (relative) BRST cohomology [42-45]:

$$
\left\langle S^{\text {phys }}\right|=\langle S| \hat{\mathcal{P}},
$$

with

$$
\mathcal{P}: \mathcal{H}_{0} \longrightarrow \mathcal{H}_{Q}, \quad \hat{\mathcal{P}}=\sum_{n=0}^{\infty} \frac{1}{n !} \underbrace{\mathcal{P} \wedge \cdots \wedge \mathcal{P}}_{n} .
$$

Decoupling of unphysical states from the physical S-matrix is guaranteed by

$$
\begin{aligned}
{\left[\boldsymbol{Q}, \boldsymbol{S}_{\mathrm{int}}\right] } & =\hat{\boldsymbol{P}}\left(\left[\boldsymbol{Q}, \boldsymbol{L}_{\mathrm{int}}\right]-\boldsymbol{L}_{\mathrm{int}}\left(\hat{\boldsymbol{I}}-\boldsymbol{H} \boldsymbol{L}_{\mathrm{int}}\right)^{-1}\left[\boldsymbol{Q}, \boldsymbol{H} \boldsymbol{L}_{\mathrm{int}}\right]\right)\left(\hat{\boldsymbol{I}}-\boldsymbol{H} \boldsymbol{L}_{\mathrm{int}}\right)^{-1} \hat{\boldsymbol{P}} \\
& =\hat{\boldsymbol{P}} \boldsymbol{L}_{\mathrm{int}}\left(\hat{\boldsymbol{I}}-\boldsymbol{H} \boldsymbol{L}_{\mathrm{int}}\right)^{-1} \hat{\boldsymbol{P}} \boldsymbol{L}_{\mathrm{int}}\left(\hat{\boldsymbol{I}}-\boldsymbol{H} \boldsymbol{L}_{\mathrm{int}}\right)^{-1} \hat{\boldsymbol{P}}=0 .
\end{aligned}
$$

The last equality follows from the fact that the internal states are generically off-shell.

Here, we have derived the S-matrix generating functional (2.54) following the physical consideration given in ref. [36]. However, it can also be obtained as an (almost) minimal model of the cyclic $L_{\infty}$ algebra $\left(\mathcal{H}^{\text {res }}, \Omega, \boldsymbol{L}\right)$ by means of HPT $[46,47]$. This alternative derivation is given in appendix A.

\subsection{Evaluation of the S-matrix}

Using the multilinear representation (2.55a), we can show that all the tree-level amplitudes of the heterotic string field theory agree with those calculated in the first-quantized formulation.

Let us define two maps on the symmetrized tensor algebra $\mathcal{S} \mathcal{H}^{\text {res: }}$

$$
\hat{\boldsymbol{i}}^{\prime}=\left(\hat{\boldsymbol{I}}-\boldsymbol{H} \boldsymbol{L}_{\mathrm{int}}\right)^{-1} \hat{\boldsymbol{P}}, \quad \boldsymbol{\Sigma}=\boldsymbol{l} \hat{\boldsymbol{i}}^{\prime} .
$$


Here, as shown in appendix A, the first map $\hat{\boldsymbol{i}}^{\prime}$ is the cohomomorphism as a deformed chain map in the HPT, and thus determined by its component $\pi_{1} \hat{\boldsymbol{i}}^{\prime}: \mathcal{S} \mathcal{H}^{\text {res }} \rightarrow \mathcal{H}^{\text {res }}$. Another map $\boldsymbol{\Sigma}$ is related with $\boldsymbol{S}_{\text {int }}$ in $(2.54 b)$ and $\langle S|$ in (2.55a) as

$$
\pi_{1} \boldsymbol{S}_{\text {int }}=\mathcal{G} P_{0} \pi_{1} \boldsymbol{\Sigma}, \quad\langle S|=\left\langle\omega_{l}\right| \xi_{0} P_{0} \otimes P_{0} \pi_{1} \boldsymbol{\Sigma}
$$

We can deduce a relation,

$$
\pi_{1} \hat{\boldsymbol{i}}^{\prime}=P_{0} \pi_{1}-Q^{+} \mathcal{G} \pi_{1} \boldsymbol{\Sigma}
$$

between two maps. Then, from the recursive relation (2.30), we have the classical DysonSchwinger equation

$$
\begin{aligned}
\pi_{1} \boldsymbol{\Sigma} & =\sum_{n=0}^{\infty} \pi_{1} \boldsymbol{B}_{n+2}\left(\frac{1}{(n+2) !}\left(\pi_{1} \hat{\boldsymbol{i}}^{\prime}+\Xi \pi_{1}^{1} \boldsymbol{\Sigma}\right)^{\wedge(n+2)}\right) \\
& =\sum_{n=0}^{\infty} \pi_{1} \boldsymbol{B}_{n+2}\left(\frac{1}{(n+2) !}\left(P_{0} \pi_{1}-\Delta \pi_{1} \boldsymbol{\Sigma}\right)^{\wedge(n+2)}\right),
\end{aligned}
$$

where

$$
\Delta=Q^{+} \mathcal{G}-\Xi \pi^{1} .
$$

We extend the $\boldsymbol{\Sigma}$ with two parameters $t$ and $s$ counting the picture number and picture number deficit, respectively, so as to satisfy the generalized Dyson-Schwinger equation

$$
\pi_{1} \boldsymbol{\Sigma}(s, t)=\sum_{n=0}^{\infty} \pi_{1} \boldsymbol{B}_{n+2}(s, t)\left(\frac{1}{(n+2) !}\left(P_{0} \pi_{1}-\Delta(s, t) \pi_{1} \boldsymbol{\Sigma}(s, t)\right)^{\wedge(n+2)}\right),
$$

where

$$
\Delta(s, t)=Q^{+} \mathcal{G}(s, t)-t \Xi \pi^{1},
$$

with $\mathcal{G}(s, t)=\pi^{0}+(t X+s) \pi^{1}$ is determined to satisfy $\Delta(0,1)=\Delta$ and

$$
\left[\boldsymbol{Q}, \pi_{1} \boldsymbol{\Sigma}(s, t)\right]=\left[\boldsymbol{\eta}, \pi_{1} \boldsymbol{\Sigma}(s, t)\right]=0 .
$$

Then, the derivatives of the extended map $\boldsymbol{\Sigma}(s, t)$ satisfy

$$
\pi_{1} \partial_{t} \boldsymbol{\Sigma}(s, t)=\left[\boldsymbol{Q}, \pi_{1} \boldsymbol{\rho}(s, t)\right], \quad \pi_{1} \partial_{s} \boldsymbol{\Sigma}(s, t)=\left[\boldsymbol{\eta}, \pi_{1} \boldsymbol{\rho}(s, t)\right],
$$

where concrete form of $\boldsymbol{\rho}(s, t)$ is not relevant for the physical S-matrix. The derivations of eqs. (2.69) and (2.70) are given in appendices $\mathrm{C}$ and $\mathrm{D}$, respectively. Combining the two equations (2.70), we can derive the key relation

$$
\pi_{1} \partial_{t} \boldsymbol{\Sigma}(s, t)-X_{0} \circ \pi_{1} \partial_{s} \boldsymbol{\Sigma}(s, t)=\left[\boldsymbol{Q},\left[\boldsymbol{\eta}, \pi_{1} \boldsymbol{T}(s, t)\right]\right],
$$

with $\pi_{1} \boldsymbol{T}(s, t)=\xi_{0} \circ \pi_{1} \boldsymbol{\rho}(s, t)$. Here, for the operator $\mathcal{O}=\xi_{0}$ or $X_{0}$, the operation $\mathcal{O} \circ$ on $\pi_{1} \boldsymbol{D}_{n}$ is defined by

$$
\mathcal{O} \circ \pi_{1} \boldsymbol{D}_{n}=\frac{1}{n+1}\left(\mathcal{O} D_{n}+(-1)^{|\mathcal{O}||D|} D_{n}\left(\mathcal{O} \pi_{1} \wedge \mathbb{I}_{n-1}\right)\right) .
$$


We can find from the definition that the extended S-matrix $\langle S(s, t)|$ defined by

$$
\langle S(s, t)|=\left\langle\omega_{l}\right| \xi_{0} P_{0} \otimes P_{0} \pi_{1} \Sigma(s, t)
$$

can be expanded as ${ }^{9}$

$$
\begin{aligned}
\langle S(s, t)| & =\sum_{n=0}^{\infty}\left\langle S_{n+3}(s, t)\right|, \\
\left\langle S_{n+3}(s, t)\right| & =\sum_{m=0}^{n+1} s^{m}\left\langle S_{n+3}^{[m]}(t)\right|, \quad\left\langle S_{n+3}^{[m]}(t)\right|=\sum_{p=0}^{n-m+1} t^{p}\left\langle\left. S_{n+3}^{(p)}\right|^{2(n-m-p+1)},\right.
\end{aligned}
$$

induced by the expansion

$$
\begin{aligned}
\boldsymbol{\Sigma}(s, t) & =\sum_{n=0}^{\infty} \boldsymbol{\Sigma}_{n+2}(s, t), \\
\boldsymbol{\Sigma}_{n+2}(s, t) & =\sum_{m=0}^{n+1} s^{m} \boldsymbol{\Sigma}_{n+2}^{[m]}(t), \quad \boldsymbol{\Sigma}_{n+2}^{[m]}(t)=\left.\sum_{p=0}^{n-m+1} t^{p} \boldsymbol{\Sigma}_{n+2}^{(p)}\right|^{2(n-m-p+1)} .
\end{aligned}
$$

The superscripts with $(\cdot)$ and $[\cdot]$ indicate the picture number and the picture number deficit, respectively. Thus, $\left\langle S_{n+3}\right|=\left\langle S_{n+3}(0,1)\right|$ is the sum of the amplitudes of several processes as

$$
\left\langle S_{n+3}\right|=\sum_{p=0}^{n+1}\left\langle\left. S_{n+3}^{(p)}\right|^{2(n-p+1)} .\right.
$$

For example, $\left\langle S_{4}\right|$ is the sum of three amplitudes

$$
\left\langle S_{4}\right|=\left\langle\left. S_{4}^{(0)}\right|^{4}+\left\langle\left. S_{4}^{(1)}\right|^{2}+\left\langle\left. S_{4}^{(2)}\right|^{0} .\right.\right.\right.
$$

The first and third terms are four-R and four-NS string scattering amplitudes, respectively. The second term is further the sum of two expressions of the two-R-two-NS scattering amplitude, depending on whether the output (of $\boldsymbol{\Sigma}$ ) is the NS or R state:

$$
\left\langle\left. S_{4}^{(1)}\right|^{2}=\left\langle\left. S_{4}^{(1)}\right|_{2} ^{2}+\left\langle\left. S_{4}^{(1)}\right|_{0} ^{2} .\right.\right.\right.
$$

Each amplitude can be calculated by solving the Dyson-Schwinger equation (2.65). We find

$$
\begin{aligned}
\left\langle\left. S_{4}^{(0)}\right|_{2} ^{4}=\left\langle\omega_{l}\right| \xi_{0} P_{0} \otimes P_{0} \pi_{1}^{1}\left(\left.\boldsymbol{B}_{3}^{(0)}\right|_{2} ^{4}-\left.\boldsymbol{B}_{2}^{(0)}\right|_{0} ^{2}\left(\left.\mathbb{I} \wedge \frac{b_{0}^{+}}{L_{0}^{+}} \pi_{1}^{0} \boldsymbol{B}_{2}^{(0)}\right|_{2} ^{2}\right)\right)\right) P_{3} \pi_{3}, \\
\left\langle\left. S_{4}^{(1)}\right|_{0} ^{2}=\left\langle\omega_{l}\right| \xi_{0} P_{0} \otimes P_{0} \pi_{1}^{1}\left(\left.\boldsymbol{B}_{3}^{(1)}\right|_{0} ^{2}-\left.\boldsymbol{B}_{2}^{(0)}\right|_{0} ^{2}\left(\left.\mathbb{I} \wedge \frac{b_{0}^{+}}{L_{0}^{+}} \pi_{1}^{0} \boldsymbol{B}_{2}^{(1)}\right|_{0} ^{0}\right)\right)\right. \\
\left.\left.-\left.\boldsymbol{B}_{2}^{(0)}\right|_{0} ^{2}\left(\left.\mathbb{I} \wedge\left(\frac{b_{0}^{+} X}{L_{0}^{+}}-\Xi\right) \pi_{1}^{0} \boldsymbol{B}_{2}^{(0)}\right|_{0} ^{2}\right)\right)\right) P_{3} \pi_{3}, \\
\left\langle\left. S_{4}^{(2)}\right|_{0} ^{0}=\left\langle\omega_{l}\right| \xi_{0} P_{0} \otimes P_{0} \pi_{1}^{0}\left(\left.\boldsymbol{B}_{3}^{(2)}\right|_{0} ^{0}-\left.\boldsymbol{B}_{2}^{(1)}\right|_{0} ^{0}\left(\left.\mathbb{I} \wedge \frac{b_{0}^{+}}{L_{0}^{+}} \pi_{1}^{0} \boldsymbol{B}_{2}^{(1)}\right|_{0} ^{0}\right)\right)\right) P_{3} \pi_{3},
\end{aligned}
$$

\footnotetext{
${ }^{9}$ Note that the lower bound of the summation with respect to $p$ is actually restricted by the constraint that the cyclic Ramond number (= No. of R-inputs + No. of R-outputs) has to be less than the number of external lines (= No. of inputs + No. of outputs).
} 
which agree with those calculated in ref. [24], and an alternative expression of the two-NStwo-R amplitude,

$$
\begin{aligned}
\left\langle\left. S_{4}^{(1)}\right|_{2} ^{2}=\left\langle\omega_{l}\right| \xi_{0} P_{0} \otimes P_{0} \pi_{1}^{0}\left(\left.\boldsymbol{B}_{3}^{(1)}\right|_{2} ^{2}-\left.\boldsymbol{B}_{2}^{(1)}\right|_{0} ^{0}(\mathbb{I}\right.\right. & \left.\left.\left.\wedge \frac{b_{0}^{+}}{L_{0}^{+}} \pi_{1}^{0} \boldsymbol{B}_{2}^{(0)}\right|_{2} ^{2}\right)\right) \\
-\left.\boldsymbol{B}_{2}^{(0)}\right|_{2} ^{2}(\mathbb{I} & \left.\left.\left.\left.\wedge\left(\frac{b_{0}^{+} X}{L_{0}^{+}}-\Xi\right) \pi_{1}^{1} \boldsymbol{B}_{2}^{(0)}\right|_{0} ^{2}\right)\right)\right) P_{3} \pi_{3} .
\end{aligned}
$$

It is not difficult to prove that the physical S-matrix agrees with that calculated in the first-quantized formulation. From (2.71) we find that the extended S-matrix satisfies the relation

$$
\partial_{t}\langle S(s, t)|-\partial_{s}\langle S(s, t)|\left(X_{0}\right)_{\mathrm{cyc}}=\left\langle\omega_{l}\right| \xi_{0} P_{0} \otimes P_{0} \pi_{1}[\boldsymbol{Q},[\boldsymbol{\eta}, \boldsymbol{T}(s, t)]],
$$

where $\langle S(s, t)|\left(X_{0}\right)_{\text {cyc }}$ is defined by

$$
\langle S(s, t)|\left(X_{0}\right)_{\mathrm{cyc}}=\sum_{n=0}^{\infty}\left\langle S_{n+3}(s, t)\right|\left(X_{0} \otimes \mathbb{I}_{n+2}+\mathbb{I} \otimes \mathbb{I}_{n+1} \wedge X_{0}\right) .
$$

Then, using the expansion in $(2.74 \mathrm{~b})$, we can find the relation between components with different picture numbers:

$$
(p+1)\left\langle\left. S_{n+3}^{(p+1)}\right|^{2(n-m-p)}-(m+1)\left\langle\left. S_{n+3}^{(p)}\right|^{2(n-m-p)}\left(X_{0}\right)_{\mathrm{cyc}}=\left\langle\omega_{l}\right| \xi_{0} P_{0} \otimes P_{0} \pi_{1}[\boldsymbol{Q},[\boldsymbol{\eta}, *]] .\right.\right.
$$

The explicit form of the right hand side is not important since it does not contribute to the physical S-matrix thanks to the fact that $\boldsymbol{Q} \hat{\mathcal{P}}=\boldsymbol{\eta} \hat{\mathcal{P}}=0$. By using this relation repeatedly, we can find the physical amplitude can eventually be written as

$$
\left\langle S_{n+3}^{\text {phys }}\right|=\sum_{p=0}^{n+1}\left\langle\left. S_{n+3}^{(0)}\right|^{2(n-p+1)}\left(\left(X_{0}\right)_{\text {cyc }}\right)^{p} \hat{\mathcal{P}} .\right.
$$

In this final form, the PCOs in an amplitude are acting on the external states in a way that is common to all the Feynman diagrams, so each amplitude is written as integral of a smooth function (section) over the whole moduli space including external states accompanied by PCOs. If we further note that the differences in PCOs we use and in the states they act can be written in the form of $[Q,[\eta, *]],{ }^{10}$ we can replace $X_{0}$ s with the local ones $X(z)$ s so that all the external NS and R states have the picture number either -1 or 0 and $-1 / 2$ or $-3 / 2$, respectively. Then, we can conclude that the heterotic string field theory reproduces the tree-level S-matrix calculated in the covariant first-quantized formulation [26].

\footnotetext{
${ }^{10}$ For example, if we map the worldsheet to the complex $z$ plane so that the string 1 and 2 are on the points $Z_{1}$ and $Z_{2}$, respectively, the differences can be written as

$$
\begin{aligned}
\left(X_{0}\right)_{1}-\left(X_{0}\right)_{2} & =\left[Q,\left[\eta, \xi_{0} \oint_{Z_{1}} \frac{d z_{1}}{2 \pi i z_{1}} \oint_{Z_{2}} \frac{d z_{2}}{2 \pi i z_{2}} \int_{z_{2}}^{z_{1}} d w \partial \xi(w)\right]\right] \\
\left(X_{0}\right)_{1}-X\left(Z_{1}\right) & =\left[Q,\left[\eta, \xi_{0} \oint_{Z_{1}} \frac{d z_{1}}{2 \pi i z_{1}} \int_{Z_{1}}^{z_{1}} d z \partial \xi(z)\right]\right]
\end{aligned}
$$
}

where $\left(X_{0}\right)_{1}$ and $\left(X_{0}\right)_{2}$ are $X_{0}$ s acting on the string 1 and 2 , respectively. 


\section{Extension to the type II superstring field theory}

In the previous section, we show that the tree-level physical S-matrix of the heterotic string field theory agrees with that calculated in the conventional first-quantized formulation. This proof is easily extended to the type II superstring field theory since it was constructed by repeating the prescription developed for the heterotic string field theory [25].

\subsection{Type II superstring field theory with $L_{\infty}$ structure}

The type II superstring field $\Phi$ is Grassmann even and has four components with ghost number 2:

$$
\Phi=\Phi_{\mathrm{NS}-\mathrm{NS}}+\Phi_{\mathrm{R}-\mathrm{NS}}+\Phi_{\mathrm{NS}-\mathrm{R}}+\Phi_{\mathrm{R}-\mathrm{R}} \in \mathcal{H}^{\mathrm{res}}=\mathcal{H}_{\mathrm{NS}-\mathrm{NS}}+\mathcal{H}_{\mathrm{R}-\mathrm{NS}}^{\mathrm{res}}++\mathcal{H}_{\mathrm{NS}-\mathrm{R}}^{\mathrm{res}}+\mathcal{H}_{\mathrm{R}-\mathrm{R}}^{\mathrm{res}}
$$

The NS-NS component $\Phi_{\text {NS-NS }}$, R-NS component $\Phi_{\mathrm{R}-\mathrm{NS}}, \mathrm{NS}-\mathrm{R}$ component $\Phi_{\mathrm{NS}-\mathrm{R}}$, and $\mathrm{R}-\mathrm{R}$ component $\Phi_{\mathrm{R}-\mathrm{R}}$ have picture numbers $(-1,-1),(-1 / 2,-1),(-1,-1 / 2)$, and $(-1 / 2,-1 / 2)$, respectively. The Hilbert space $\mathcal{H}^{\text {res }}$ is restricted by the closed string constraints (2.1) and an extra condition (2.8) with

$$
\begin{aligned}
& \mathcal{G}=\mathscr{X} \overline{\mathscr{X}}, \quad \mathscr{X}=\pi^{0}+X \pi^{1}, \quad \overline{\mathscr{X}}=\bar{\pi}^{0}+\bar{X} \bar{\pi}^{1}, \\
& \mathcal{G}^{-1}=\mathscr{Y} \overline{\mathscr{Y}}, \quad \mathscr{Y}=\pi^{0}+\mathcal{Y} \pi^{1}, \quad \overline{\mathscr{Y}}=\bar{\pi}^{0}+\overline{\mathcal{Y}} \bar{\pi}^{1},
\end{aligned}
$$

where $\pi^{0}$ and $\pi^{1}\left(\bar{\pi}^{0}\right.$ and $\left.\bar{\pi}^{1}\right)$ are the projection operators onto the NS and R states in the holomorphic (anti-holomorphic) sectors, respectively. We take the non-local operators

$$
\mathcal{Y}=-2 \frac{G}{L_{0}^{+}} \delta\left(\gamma_{0}\right), \quad \overline{\mathcal{Y}}=-2 \frac{\bar{G}}{L_{0}^{+}} \delta\left(\bar{\gamma}_{0}\right)
$$

satisfying the same relations as those satisfied by $Y$ and $\bar{Y}$,

$$
X \mathcal{Y} X=X, \quad \mathcal{Y} X \mathcal{Y}=\mathcal{Y}, \quad \bar{X} \overline{\mathcal{Y}} \bar{X}=\bar{X}, \quad \overline{\mathcal{Y}} \bar{X} \overline{\mathcal{Y}}=\overline{\mathcal{Y}},
$$

as inverse picture changing operators, which is necessary to consistently impose all the constraints. ${ }^{11}$ The constraint (2.8) can now be split into two conditions

$$
\mathscr{X} \mathscr{Y} \Phi=\overline{\mathscr{X}} \overline{\mathscr{Y}} \Phi=\Phi,
$$

which we use later. Any sate $\mathcal{B} \in \mathcal{H}^{\text {res }}$ can be expanded in the ghost zero-mode as

$$
\begin{aligned}
\mathcal{B}= & \mathcal{B}_{\mathrm{NS}-\mathrm{NS}}+\mathcal{B}_{\mathrm{R}-\mathrm{NS}}+\mathcal{B}_{\mathrm{NS}-\mathrm{R}}+\mathcal{B}_{\mathrm{R}-\mathrm{R}} \\
= & \left(b_{\mathrm{NS}}-c_{0}^{+} B_{\mathrm{NS}-\mathrm{NS}}\right)+\left(b_{\mathrm{R}-\mathrm{NS}}-\frac{1}{2}\left(\gamma_{0}+2 c_{0}^{+} G\right) B_{\mathrm{R}-\mathrm{NS}}\right) \\
& +\left(b_{\mathrm{NS}-\mathrm{R}}-\frac{1}{2}\left(\bar{\gamma}_{0}+2 c_{0}^{+} \bar{G}\right) B_{\mathrm{NS}-\mathrm{R}}\right)+\left(b_{\mathrm{R}-\mathrm{R}}-\frac{1}{2}\left(\gamma_{0} \bar{G}-\bar{\gamma}_{0} G+2 c_{0}^{+} G \bar{G}\right) B_{\mathrm{R}-\mathrm{R}}\right),
\end{aligned}
$$

\footnotetext{
${ }^{11}$ In ref. [25], we took these non-local inverse changing operators only for the R-R sector, which is enough for consistency. The result is the same in both cases if we expand the string field in the ghost zero-modes as in eq. (3.7).
} 
so we denote the string field $\Phi \in \mathcal{H}^{\text {res }}$ as

$$
\begin{aligned}
\Phi= & \Phi_{\mathrm{NS}-\mathrm{NS}}+\Phi_{\mathrm{R}-\mathrm{NS}}+\Phi_{\mathrm{NS}-\mathrm{R}}+\Phi_{\mathrm{R}-\mathrm{R}} \\
= & \left(\phi_{\mathrm{NS}}-c_{0}^{+} \psi_{\mathrm{NS}-\mathrm{NS}}\right)+\left(\phi_{\mathrm{R}-\mathrm{NS}}-\frac{1}{2}\left(\gamma_{0}+2 c_{0}^{+} G\right) \psi_{\mathrm{R}-\mathrm{NS}}\right) \\
& +\left(\phi_{\mathrm{NS}-\mathrm{R}}-\frac{1}{2}\left(\bar{\gamma}_{0}+2 c_{0}^{+} \bar{G}\right) \psi_{\mathrm{NS}-\mathrm{R}}\right)+\left(\phi_{\mathrm{R}-\mathrm{R}}-\frac{1}{2}\left(\gamma_{0} \bar{G}-\bar{\gamma}_{0} G+2 c_{0}^{+} G \bar{G}\right) \psi_{\mathrm{R}-\mathrm{R}}\right) .
\end{aligned}
$$

The on-shell subspace $\mathcal{H}_{0} \subset \mathcal{H}^{\text {res }}$ is defined by

$$
\mathcal{H}_{0}=\left\{\Phi \in \mathcal{H}^{\mathrm{res}} \mid L_{0}^{+} \Phi_{\mathrm{NS}-\mathrm{NS}}=G_{0} \Phi_{\mathrm{R}-\mathrm{NS}}=\bar{G}_{0} \Phi_{\mathrm{NS}-\mathrm{R}}=G_{0} \Phi_{\mathrm{R}-\mathrm{R}}=\bar{G}_{0} \Phi_{\mathrm{R}-\mathrm{R}}=0\right\} .
$$

The natural symplectic forms $\Omega, \omega_{s}$, and $\omega_{l}$ in the $\mathcal{H}^{\mathrm{res}}=\mathcal{H}_{L}^{\mathrm{res}} \otimes \mathcal{H}_{R}^{\mathrm{res}}, \mathcal{H}_{s}=\left(\mathcal{H}_{s}\right)_{L} \otimes\left(\mathcal{H}_{s}\right)_{R}$, and $\mathcal{H}_{l}=\left(\mathcal{H}_{l}\right)_{L} \otimes\left(\mathcal{H}_{l}\right)_{R}$, respectively, are defined similarly to those in eqs. (2.11), and are related for $\Phi_{1}, \Phi_{2} \in \mathcal{H}^{\text {res }}$ as

$$
\Omega\left(\Phi_{1}, \Phi_{2}\right)=\omega_{s}\left(\Phi_{1}, \mathcal{G}^{-1} \Phi_{2}\right)=\omega_{l}\left(\xi_{0} \bar{\xi}_{0} \Phi_{1}, \mathcal{G}^{-1} \Phi_{2}\right)
$$

It is also useful to introduce $\Omega_{m}$ in the restricted medium Hilbert space $\mathcal{H}_{m}^{\mathrm{res}}=\left(\mathcal{H}_{l}\right)_{L} \otimes \mathcal{H}_{R}^{\mathrm{res}}$ and $\omega_{m}$ in the medium Hilbert space $\mathcal{H}_{m}=\left(\mathcal{H}_{l}\right)_{L} \otimes\left(\mathcal{H}_{s}\right)_{R}$, and their counterparts the holomorphic and anti-holomorphic sectors exchanged $\Omega_{\bar{m}}$ in $\mathcal{H}_{\bar{m}}^{\text {res }}=\mathcal{H}_{L}^{\text {res }} \otimes\left(\mathcal{H}_{l}\right)_{R}$ and $\omega_{\bar{m}}$ in $\mathcal{H}_{\bar{m}}=\left(\mathcal{H}_{s}\right)_{L} \otimes\left(\mathcal{H}_{l}\right)_{R}$, which are defined in an obvious way. They are related with $\omega_{l}$ as

$$
\begin{array}{ll}
\Omega_{m}\left(\Phi_{1}, \Phi_{2}\right)=\omega_{m}\left(\Phi_{1}, \overline{\mathscr{Y}} \Phi_{2}\right)=\omega_{l}\left(\overline{\xi_{0}} \Phi_{1}, \overline{\mathscr{Y}} \Phi_{2}\right), & \Phi_{1}, \Phi_{2} \in \mathcal{H}_{m}^{\mathrm{res}}, \\
\Omega_{\bar{m}}\left(\Phi_{1}, \Phi_{2}\right)=\omega_{\bar{m}}\left(\Phi_{1}, \mathscr{Y} \Phi_{2}\right)=\omega_{l}\left(\xi_{0} \Phi_{1}, \mathscr{Y} \Phi_{2}\right), & \Phi_{1}, \Phi_{2} \in \mathcal{H}_{\bar{m}}^{\mathrm{res}},
\end{array}
$$

and with $\Omega$ as

$$
\Omega_{m}\left(\xi_{0} \Phi_{1}, \mathscr{Y} \Phi_{2}\right)=\Omega_{\bar{m}}\left(\overline{\xi_{0}} \Phi_{1}, \overline{\mathscr{Y}} \Phi_{2}\right)=\Omega\left(\Phi_{1}, \Phi_{2}\right), \quad \Phi_{1}, \Phi_{2} \in \mathcal{H}^{\text {res }}
$$

The action and the gauge transformation have the same form as those of the heterotic string field theory,

$$
\begin{aligned}
I[\Phi] & =\sum_{n=0}^{\infty} \frac{1}{(n+2) !} \Omega\left(\Phi, L_{n+1}(\underbrace{\Phi, \cdots, \Phi}_{n+1})\right), \\
\delta \Phi & =\sum_{n=0}^{\infty} \frac{1}{n !} L_{n+1}(\underbrace{\Phi, \cdots, \Phi}_{n}, \Lambda),
\end{aligned}
$$

except that the symplectic form $\Omega$ and the string products $L_{n+1}$ are now those for the type II superstring field theory.

The string products are constructed by repeating twice the prescription developed for the heterotic string products. We first apply it to the holomorphic sector and obtain the heterotic product $\boldsymbol{L}_{H}=\boldsymbol{Q}+\boldsymbol{L}_{H}^{\text {int }}$ with

$$
\begin{aligned}
\boldsymbol{L}_{H} & =\hat{\boldsymbol{F}}^{-1}\left(\boldsymbol{Q}+\pi^{0} \boldsymbol{B}\right) \hat{\boldsymbol{F}}, \\
n \pi_{1} \boldsymbol{L}_{H}^{\text {int }} & =\mathscr{X} \pi_{1} \boldsymbol{l}_{H}, \quad \pi_{1} \boldsymbol{l}_{H}=\pi_{1} \boldsymbol{B} \hat{\boldsymbol{F}} .
\end{aligned}
$$


All the quantities and relations in this first step have the same form as those of the heterotic string field theory in appearance. Next, in the second step, we repeat the prescription for the anti-holomorphic sector and construct the type II superstring product as

$$
\begin{aligned}
\boldsymbol{L} & =\hat{\boldsymbol{F}}^{-1}\left(\boldsymbol{Q}+\bar{\pi}^{0} \overline{\boldsymbol{B}}\right) \hat{\boldsymbol{F}}, \\
\pi_{1} \boldsymbol{L}_{\mathrm{int}} & =\overline{\mathscr{X}} \pi_{1} \overline{\boldsymbol{l}}, \quad \pi_{1} \overline{\boldsymbol{l}}=\pi_{1} \overline{\boldsymbol{B}} \hat{\boldsymbol{F}} .
\end{aligned}
$$

The product $\overline{\boldsymbol{B}}$ is obtained from the generating function

$$
\overline{\boldsymbol{B}}(s, t)=\left.\sum_{\bar{m}, \bar{n}, \bar{r}=0}^{\infty} s^{\bar{m}} t^{\bar{n}} \overline{\boldsymbol{B}}_{\bar{m}+\bar{n}+\bar{r}+1}^{(\bar{n})}\right|^{2 \bar{r}} \equiv \sum_{\bar{n}=0}^{\infty} t^{\bar{n}} \overline{\boldsymbol{B}}^{(\bar{n})}(s),
$$

with the gauge product

$$
\bar{\lambda}(s, t)=\left.\sum_{\bar{m}, \bar{n}, \bar{r}=0}^{\infty} s^{\bar{m}} t^{\bar{n}} \bar{\lambda}_{\bar{m}+\bar{n}+\bar{r}+2}^{(\bar{n}+1)}\right|^{2 \bar{r}}
$$

by solving the differential equation

$$
\begin{aligned}
\partial_{t} \overline{\boldsymbol{B}}(s, t) & =[\boldsymbol{Q}, \overline{\boldsymbol{\lambda}}(s, t)]+[\overline{\boldsymbol{B}}(s, t), \overline{\boldsymbol{\lambda}}(s, t)]^{\overline{1}}+s[\overline{\boldsymbol{B}}(s, t), \overline{\boldsymbol{\lambda}}(s, t)]^{\overline{2}}, \\
\partial_{s} \overline{\boldsymbol{B}}(s, t) & =[\overline{\boldsymbol{\eta}}, \overline{\boldsymbol{\lambda}}(s, t)]-t[\overline{\boldsymbol{B}}(s, t), \overline{\boldsymbol{\lambda}}(s, t)]^{\overline{2}},
\end{aligned}
$$

with the initial condition

$$
\overline{\boldsymbol{B}}^{(\overline{0})}(s)=\boldsymbol{L}_{H}^{\mathrm{int}}(s)=\left.\sum_{\bar{m}, \bar{r}=0}^{\infty} s^{\bar{m}}\left(\boldsymbol{L}_{H}\right)_{\bar{m}+\bar{r}+1}\right|^{2 \bar{r}} .
$$

The cohomomorphism $\hat{\overline{\boldsymbol{F}}}$ is given by

$$
\hat{\overline{\boldsymbol{F}}}^{-1}=\pi_{1} \mathbb{I}-\bar{\Xi} \bar{\pi}_{1}^{1} \overline{\boldsymbol{B}},
$$

where $\bar{\pi}_{1}^{1}=\pi_{1} \bar{\pi}^{1}$. Here, the picture and cyclic Ramond numbers are those of the antiholomorphic sector, which are made clear by letters with bar. The commutators $[\cdot, \cdot]^{\overline{1}, \overline{2}}$ are the analogues of $[\cdot, \cdot]^{1,2}$ projected by the cyclic Ramond number of the anti-holomorphic sector. It would be obvious that $\overline{\boldsymbol{B}}(s, t)$ satisfies the similar relations to eqs. $(2.24)$ as

$$
\begin{aligned}
{[\boldsymbol{Q}, \overline{\boldsymbol{B}}(s, t)]+\frac{1}{2}[\overline{\boldsymbol{B}}(s, t), \overline{\boldsymbol{B}}(s, t)]^{\overline{1}}+\frac{s}{2}[\overline{\boldsymbol{B}}(s, t), \overline{\boldsymbol{B}}(s, t)]^{\overline{2}}=0, } \\
{[\overline{\boldsymbol{\eta}}, \overline{\boldsymbol{B}}(s, t)]-\frac{t}{2}[\overline{\boldsymbol{B}}(s, t), \overline{\boldsymbol{B}}(s, t)]^{2}=0, }
\end{aligned}
$$

which reduce to the $L_{\infty}$ relation of the coderivation $\boldsymbol{Q}-\overline{\boldsymbol{\eta}}+\overline{\boldsymbol{B}}$ at $(s, t)=(0,1)$. If you look carefully the differential equation (3.19) and note that the initial heterotic product has the form of $\boldsymbol{L}_{H}^{\mathrm{int}}=\mathscr{X} \boldsymbol{l}_{H}$, it is found that the final form of $\overline{\boldsymbol{B}}$ has the form of

$$
\pi_{1} \overline{\boldsymbol{B}}=\mathscr{X} \pi_{1} \overline{\boldsymbol{b}}
$$

and thus $\pi_{1} \boldsymbol{L}_{\text {int }}$ is written as the form in which it is manifest that the products are closed in $\mathcal{H}^{\text {res: }}$

$$
\pi_{1} \boldsymbol{L}_{\mathrm{int}}=\mathcal{G} \pi_{1} \boldsymbol{l}, \quad \pi_{1} \boldsymbol{l}=\pi_{1} \overline{\boldsymbol{b}} \hat{\boldsymbol{F}}
$$




\subsection{S-matrix generating function and its evaluation}

Since the equation of motion has the same form as that of the heterotic string field theory, the S-matrix generating function can also be written in the same form as $(2.55 \mathrm{a})$ with

$$
\boldsymbol{S}_{\mathrm{int}}=\hat{\boldsymbol{P}} \boldsymbol{L}_{\mathrm{int}}\left(\hat{\boldsymbol{I}}-\boldsymbol{H} \boldsymbol{L}_{\mathrm{int}}\right)^{-1} \hat{\boldsymbol{P}}
$$

where $\boldsymbol{L}_{\text {int }}$ is the type II superstring product given by (3.24). By repeating the proof for the heterotic string amplitudes, we can again show that the physical amplitudes of type II superstring are obtained as integrals of the smooth functions (sections) over the whole moduli space with appropriate picture changed external states.

Let us briefly summarize the procedure for the type II superstring. If we define the maps

$$
\hat{\boldsymbol{i}}^{\prime}=\left(\hat{\boldsymbol{I}}-\boldsymbol{H} \boldsymbol{L}_{\mathrm{int}}\right)^{-1} \hat{\boldsymbol{P}}, \quad \pi_{1} \overline{\boldsymbol{\Sigma}}=\pi_{1} \hat{\boldsymbol{l}}^{\hat{\boldsymbol{i}}^{\prime}},
$$

the S-matrix can be represented by $\overline{\boldsymbol{\Sigma}}$ similar to that of the heterotic string field theory as

$$
\begin{aligned}
\pi_{1} \boldsymbol{S}_{\mathrm{int}} & =\overline{\mathscr{X}} P_{0} \pi_{1} \overline{\boldsymbol{\Sigma}}, \\
\langle S| & =\langle\Omega| P_{0} \otimes \pi_{1} \boldsymbol{S}_{\mathrm{int}}=\left\langle\Omega_{\bar{m}}\right| \bar{\xi}_{0} P_{0} \otimes P_{0} \pi_{1} \overline{\boldsymbol{\Sigma}} .
\end{aligned}
$$

The $\bar{\Sigma}$ is recursively determined by the equation

$$
\pi_{1} \bar{\Sigma}=\sum_{n=0}^{\infty} \pi_{1} \overline{\boldsymbol{B}}_{n+2}\left(\frac{1}{(n+2) !}\left(P_{0} \pi_{1}-\bar{\Delta} \pi_{1} \boldsymbol{\Sigma}\right)^{\wedge(n+2)}\right),
$$

with

$$
\bar{\Delta}=Q^{+} \overline{\mathscr{X}}-\bar{\Xi} \bar{\pi}^{1} .
$$

If we define two parameter extension $\overline{\boldsymbol{\Sigma}}(s, t)$ using $\overline{\boldsymbol{B}}(s, t)$ and

$$
\bar{\Delta}(s, t)=Q^{+} \overline{\mathscr{X}}(s, t)-t \bar{\Xi} \bar{\pi}^{1}, \quad \overline{\mathscr{X}}(s, t)=\bar{\pi}^{0}+(t \bar{X}+s) \bar{\pi}^{1},
$$

it is easy to see that the relations

$$
\partial_{t} \overline{\boldsymbol{\Sigma}}(s, t)=[\boldsymbol{Q}, \overline{\boldsymbol{\rho}}(s, t)], \quad \partial_{s} \overline{\boldsymbol{\Sigma}}(s, t)=[\overline{\boldsymbol{\eta}}, \overline{\boldsymbol{\rho}}(s, t)],
$$

hold similar to the case of the heterotic string field theory. The explicit form of $\overline{\boldsymbol{\rho}}(s, t)$ is not relevant for the physical S-matrix, but obtained similarly to that of the heterotic string given in appendix D. The relations (3.32) provides the key equation

$$
\partial_{t} \overline{\boldsymbol{\Sigma}}(s, t)-\bar{X}_{0} \circ \partial_{s} \overline{\boldsymbol{\Sigma}}(s, t)=[\boldsymbol{Q},[\overline{\boldsymbol{\eta}}, \overline{\boldsymbol{T}}(s, t)]],
$$

with $\overline{\boldsymbol{T}}(s, t)=\bar{\xi}_{0} \circ \overline{\boldsymbol{\rho}}(s, t)$. Then, the extended S-matrix

$$
\left\langle S_{n+3}(s, t)\right|=\sum_{\bar{m}=0}^{n+1} s^{\bar{m}}\left\langle S_{n+3}^{[\bar{m}]}(t)\right|, \quad\left\langle S_{n+3}^{[\bar{m}]}(t)\right|=\sum_{\bar{p}=0}^{n-\bar{m}+1} t^{\bar{p}}\left\langle\left. S_{n+3}^{(\bar{p})}\right|^{2(n-\bar{m}-\bar{p}+1)},\right.
$$

induced by the expansion

$$
\overline{\boldsymbol{\Sigma}}_{n+2}(s, t)=\sum_{\bar{m}=0}^{n+1} s^{\bar{m}} \overline{\boldsymbol{\Sigma}}_{n+2}^{[\bar{m}]}(t), \quad \overline{\boldsymbol{\Sigma}}_{n+2}^{[\bar{m}]}(t)=\left.\sum_{\bar{p}=0}^{n-\bar{m}+1} t^{\bar{p}} \overline{\boldsymbol{\Sigma}}_{n+2}^{(\bar{p})}\right|^{2(n-\bar{m}-\bar{p}+1)},
$$


satisfies the relation

$$
\partial_{t}\langle S(s, t)|-\partial_{s}\langle S(s, t)| \bar{X}_{0}=\left\langle\Omega_{m}\right| \bar{\xi}_{0} P_{0} \otimes P_{0} \pi_{1}[\boldsymbol{Q},[\overline{\boldsymbol{\eta}}, \overline{\boldsymbol{T}}(s, t)]] .
$$

Hence, for the physical S-matrix, we have

$$
\left\langle S_{n+3}^{\text {phys }}\right|=\sum_{\bar{p}=0}^{n+1}\left\langle\left. S_{n+3}^{(\overline{0})}\right|^{2(n-\bar{p}+1)}\left(\bar{X}_{0}\right)^{\bar{p}} \hat{\mathcal{P}},\right.
$$

in a similar way to the case of the heterotic string field theory. Here, $\hat{\mathcal{P}}$ is the projection operator onto the physical Hilbert space satisfying $\boldsymbol{Q} \hat{\mathcal{P}}=\boldsymbol{\eta} \hat{\mathcal{P}}=\overline{\boldsymbol{\eta}} \hat{\mathcal{P}}=0$. The amplitudes with no anti-holomorphic picture number $\left\langle S_{n+3}^{(\overline{0})}\right|$ is nothing but those of the heterotic string field theory,

$$
\left\langle S_{n+3}^{(\overline{0})}\right|=\left\langle\left(S_{H}\right)_{n+3}\right|,
$$

by construction. It is embedded in the type II superstring Hilbert space as

$$
\left\langle S_{H}\right|=\left\langle\Omega_{m}\right| \xi_{0} P_{0} \otimes \mathscr{Y} \pi_{1} \boldsymbol{S}_{H}=\left\langle\Omega_{m}\right| \xi_{0} P_{0} \otimes P_{0} \pi_{1} \boldsymbol{\Sigma}_{H},
$$

with

$$
\boldsymbol{S}_{H}=\hat{\boldsymbol{P}} \boldsymbol{L}_{H}^{\mathrm{int}}\left(\hat{\boldsymbol{I}}-\boldsymbol{H} \boldsymbol{L}_{H}^{\mathrm{int}}\right)^{-1} \hat{\boldsymbol{P}}
$$

So, now it is easy to see that

$$
\left\langle\left(S_{H}\right)_{n+3}\right|=\sum_{p=0}^{n+1}\left\langle\left. S_{n+3}^{(0)}\right|^{2(r-p+1)}\left(X_{0}\right)^{p},\right.
$$

without explicitly repeating the procedure again. Substituting this into the first step result (3.37), the physical S-matrix of the type II superstring field theory can be written as

$$
\left\langle S_{n+3}^{\text {phys }}\right|=\sum_{p=0}^{n+1} \sum_{\bar{p}=0}^{n+1}\left\langle\left. S_{n+3}^{(0, \overline{0})}\right|^{(2(n-p+1), 2(n-\bar{p}+1))}\left(X_{0}\right)^{p}\left(\bar{X}_{0}\right)^{\bar{p}} \hat{\mathcal{P}} .\right.
$$

From the similar consideration to that in the heterotic string field theory, this agrees with the tree-level physical $(n+3)$-string scattering amplitudes obtained in the covariant firstquantized formulation.

\section{Extension to the open superstring field theory}

The proof given for closed superstring theories in the previous sections can also be applied to the case of the open superstring field theory. In this section, we first extend our construction method to the open superstring field theory, and then, prove that it reproduces the wellknown tree-level S-matrix. 


\subsection{Open superstring field theory with cyclic $A_{\infty}$ structure}

The open superstring field $\Phi$ is Grassmann odd and has two components with ghost number 1:

$$
\Phi=\Phi_{\mathrm{NS}}+\Phi_{R} \in \mathcal{H}^{\mathrm{res}}=\mathcal{H}_{\mathrm{NS}}+\mathcal{H}_{R}^{\mathrm{res}}
$$

The NS component $\Phi_{\mathrm{NS}}$ and R component $\Phi_{R}$ have picture numbers -1 and $-1 / 2$, respectively. The Hilbert space $\mathcal{H}^{\text {res }}$ is restricted by a constraint (2.8) with

$$
\begin{aligned}
\mathcal{G} & =\pi^{0}+X \pi^{1}, & & X=-\delta\left(\beta_{0}\right) G_{0}+\delta^{\prime}\left(\beta_{0}\right) b_{0}, \\
\mathcal{G}^{-1} & =\pi^{0}+Y \pi^{1}, & & Y=-c_{0} \delta^{\prime}\left(\gamma_{0}\right) .
\end{aligned}
$$

The string field $\Phi \in \mathcal{H}^{\text {res }}$ is expanded in the ghost zero-modes as

$$
\Phi=\left(\phi_{\mathrm{NS}}-c_{0} \psi_{\mathrm{NS}}\right)+\left(\phi_{R}-\frac{1}{2}\left(\gamma_{0}+c_{0} G\right) \psi_{R}\right) .
$$

The on-shell subspace $\mathcal{H}_{0} \subset \mathcal{H}^{\text {res }}$ and the projector $P_{0}$ are introduced as

$$
P_{0}: \mathcal{H}^{\mathrm{res}} \longrightarrow \mathcal{H}_{0}=\left\{\Phi \in \mathcal{H}^{\mathrm{res}} \mid L_{0} \Phi_{\mathrm{NS}}=G_{0} \Phi_{R}=0\right\}
$$

The symplectic forms are defined by

$$
\begin{aligned}
\omega_{l}\left(\Phi_{1}, \Phi_{2}\right) & =(-1)^{\operatorname{deg}\left(\Phi_{1}\right)}{ }_{l}\left\langle\Phi_{1} \mid \Phi_{2}\right\rangle_{l}, & & \Phi_{1}, \Phi_{2} \in \mathcal{H}_{l}, \\
\omega_{s}\left(\Phi_{1}, \Phi_{2}\right) & =(-1)^{\operatorname{deg}\left(\Phi_{1}\right)}{ }_{s}\left\langle\Phi_{1} \mid \Phi_{2}\right\rangle_{s}, & & \Phi_{1}, \Phi_{2} \in \mathcal{H}_{s}, \\
\Omega\left(\Phi_{1}, \Phi_{2}\right) & =(-1)^{\operatorname{deg}\left(\Phi_{1}\right)}{ }_{s}\left\langle\Phi_{1}\left|\mathcal{G}^{-1}\right| \Phi_{2}\right\rangle_{s}, & & \Phi_{1}, \Phi_{2} \in \mathcal{H}^{\mathrm{res}}
\end{aligned}
$$

using the BPZ inner product. The degree of generic states $\Phi_{1,2}$ are defined by $\operatorname{deg}\left(\Phi_{1,2}\right)=$ $\left(\left|\Phi_{1,2}\right|+1\right) \bmod 2$, and thus, in particular, the degree of the open superstring field $\Phi$ is even.

The action and gauge transformation are now given by

$$
I[\Phi]=\sum_{n=0}^{\infty} \frac{1}{n+2} \Omega\left(\Phi, M_{n+1}(\underbrace{\Phi, \cdots, \Phi}_{n+1})\right),
$$

and

$$
\delta \Phi=\sum_{n=0}^{\infty} \sum_{k=0}^{n} M_{n+1}(\underbrace{\Phi, \cdots, \Phi}_{n-k}, \Lambda, \underbrace{\Phi, \cdots, \Phi}_{k}),
$$

by using the non-commutative open-superstring products, $M_{1}=Q$ and $M_{n+2}(n \geq 0)$, which satisfy the $A_{\infty}$ relations

$$
\begin{aligned}
& \sum_{m=0}^{n} \sum_{j=0}^{n-m}(-1)^{\epsilon(1, j)} \\
& \quad \times M_{n-m+1}\left(\Phi_{1}, \cdots, \Phi_{j}, M_{m+1}\left(\Phi_{j+1}, \cdots, \Phi_{j+m+1}\right), \Phi_{j+m+2}, \cdots, \Phi_{n+1}\right)=0,
\end{aligned}
$$


and the cyclicity condition

$$
\Omega\left(M_{n}\left(\Phi_{1}, \cdots, \Phi_{n}\right), \Phi_{n+1}\right)=(-1)^{\operatorname{deg}\left(\Phi_{1}\right) \epsilon(2, n+1)} \Omega\left(M_{n}\left(\Phi_{2}, \cdots, \Phi_{n+1}\right), \Phi_{1}\right),
$$

where $\epsilon(i, j)=\sum_{k=i}^{j} \operatorname{deg}\left(\Phi_{k}\right)$.

Our construction method of the string products of the heterotic string field theory is also applicable to those of the open superstring field theory. It is achieved by simply replacing the coderivation $\boldsymbol{B}(s, t)$ acting on the symmetrized tensor algebra $\mathcal{S H}_{l}$ with the coderivation

$$
\boldsymbol{A}(s, t)=\left.\sum_{n, m, r=0}^{\infty} s^{m} t^{n} \boldsymbol{A}_{m+n+r+1}^{(n)}\right|^{2 r},
$$

acting on the tensor algebra $\mathcal{T} \mathcal{H}^{\text {res }}=\oplus_{n=0}^{\infty}\left(\mathcal{H}^{\text {res }}\right)^{\otimes n}$. If $\boldsymbol{A}(s, t)$ satisfies the differential equations

$$
\begin{aligned}
\partial_{t} \boldsymbol{A}(s, t) & =[\boldsymbol{Q}, \boldsymbol{\mu}(s, t)]+[\boldsymbol{A}(s, t), \boldsymbol{\mu}(s, t)]^{1}+s[\boldsymbol{A}(s, t), \boldsymbol{\mu}(s, t)]^{2}, \\
\partial_{s} \boldsymbol{A}(s, t) & =[\boldsymbol{\eta}, \boldsymbol{\mu}(s, t)]-t[\boldsymbol{A}(s, t), \boldsymbol{\mu}(s, t)]^{2},
\end{aligned}
$$

with the (even) coderivation of the open superstring gauge products

$$
\boldsymbol{\mu}(s, t)=\left.\sum_{m, n, r=0}^{\infty} s^{m} t^{n} \boldsymbol{\mu}_{m+n+r+2}^{(n+1)}\right|^{2 r}
$$

it satisfies the similar relations to eqs. (2.24),

$$
\begin{aligned}
{[\boldsymbol{Q}, \boldsymbol{A}(s, t)]+\frac{1}{2}[\boldsymbol{A}(s, t), \boldsymbol{A}(s, t)]^{1}+\frac{s}{2}[\boldsymbol{A}(s, t), \boldsymbol{A}(s, t)]^{2} } & =0, \\
{[\boldsymbol{\eta}, \boldsymbol{A}(s, t)]-\frac{t}{2}[\boldsymbol{A}(s, t), \boldsymbol{A}(s, t)]^{2} } & =0 .
\end{aligned}
$$

At $(s, t)=(0,1)$, they reduce to the $A_{\infty}$ relations of the coderivation $\boldsymbol{Q}-\boldsymbol{\eta}+\boldsymbol{A}(0,1)$. All the open superstring products, and simultaneously the gauge products, are determined in the same way as those of the heterotic string field theory by solving the differential equations in (4.11) starting from the initial condition

$$
\boldsymbol{A}(s, 0)=\boldsymbol{M}_{B}^{(0)}(s)=\left.\sum_{m, r=0}^{\infty} s^{m} \boldsymbol{M}_{m+r+1}^{(0)}\right|^{2 r} .
$$

Here, $\boldsymbol{M}_{n+2}^{(0)}$ are the open string products without PCO-insertions, which we call the open bosonic string products. The cyclic $A_{\infty}$ algebra $\boldsymbol{M}=\boldsymbol{Q}+\boldsymbol{M}_{\text {int }}$ is constructed from $\boldsymbol{A}=\boldsymbol{A}(0,1)$ as

$$
\begin{aligned}
\boldsymbol{M} & =\hat{\boldsymbol{F}}^{-1}\left(\boldsymbol{Q}+\pi^{0} \boldsymbol{A}\right) \hat{\boldsymbol{F}}, \\
\pi_{1} \boldsymbol{M}_{\mathrm{int}} & =\mathcal{G} \boldsymbol{a}, \quad \pi_{1} \boldsymbol{a}=\pi_{1} \boldsymbol{A} \hat{\boldsymbol{F}},
\end{aligned}
$$

using the cohomomorphism

$$
\pi_{1} \hat{\boldsymbol{F}}^{-1}=\pi_{1} \mathbb{I}-\Xi \pi_{1}^{1} \boldsymbol{A}
$$


This construction is an extension of that in ref. [9] proposed for the products in the NS sector. If we restrict our construction to the NS sector, the cohomomorphism $\hat{\boldsymbol{F}}$ becomes trivial, $\pi_{1}^{0} \hat{\boldsymbol{F}}=\pi_{1}^{0} \mathbb{I}$, and the differential equations (4.11) reduce to those in ref. [9] for the string product

$$
\left.\boldsymbol{A}\right|^{0}(s, t)=\left.\sum_{n, m=0}^{\infty} s^{m} t^{n} \boldsymbol{A}_{n+m+1}^{(n)}\right|^{0} .
$$

It is also a generalization of that in ref. [17], in which a complete action of the open superstring field theory based on the $A_{\infty}$ algebra was first constructed. The string products with $A_{\infty}$ structure (4.15) completely agree with those constructed in ref. [17] if we take the associative bosonic product, $\boldsymbol{M}_{B}^{(0)}(s)=\left.\boldsymbol{m}_{2}\right|^{0}+\left.s \boldsymbol{m}_{2}\right|^{2}$, as an initial condition, ${ }^{12}$ which is explicitly in appendix E. Our construction method, however, allows us to take more general initial bosonic products, and provides more general superstring products which were not constructed before. This degree of freedom allows for the various open string field theories realizing different triangulations of the moduli space, which will be useful in analyzing specific problems. The proof of cyclicity becomes easier in our construction, which we give in appendix F.

\subsection{S-matrix generating function and its evaluation}

The tree-level S-matrix generating functional is obtained in a similar way to the heterotic string field theory:

$$
\langle S|=\langle\Omega| P_{0} \otimes \pi_{1} S_{\text {int }}: \mathcal{H}_{0} \otimes \mathcal{T} \mathcal{H}_{0} \longrightarrow \mathbb{C},
$$

with

$$
\boldsymbol{S}_{\mathrm{int}}=\hat{\boldsymbol{P}} \boldsymbol{M}_{\mathrm{int}}\left(\boldsymbol{I}-\boldsymbol{H} \boldsymbol{M}_{\text {int }}\right)^{-1} \hat{\boldsymbol{P}} .
$$

Here, all the quantities are those acting on $\mathcal{T} \mathcal{H}^{\text {res }}$, which are similarly defined for the open superstring,

$$
\boldsymbol{H}=\sum_{r, s=0}^{\infty} \mathbb{I}^{\otimes r} \otimes\left(-Q^{+}\right) \otimes P_{0}^{\otimes s}, \quad \hat{\boldsymbol{P}}=\sum_{n=0}^{\infty} P_{0}^{\otimes n}, \quad \hat{\boldsymbol{I}}=\sum_{n=0}^{\infty} \mathbb{I}^{\otimes n},
$$

using the open string homotopy operator

$$
Q^{+}=\frac{1}{L_{0}} b_{0}\left(1-P_{0}\right),
$$

and satisfy the same relation as (2.50). If we introduce two maps

$$
\hat{\boldsymbol{i}}^{\prime}=\left(\hat{\boldsymbol{I}}-\boldsymbol{H} \boldsymbol{M}_{\mathrm{int}}\right)^{-1} \hat{\boldsymbol{P}}, \quad \boldsymbol{\Sigma}=\boldsymbol{a} \hat{\boldsymbol{i}}^{\prime},
$$

the map $\boldsymbol{\Sigma}$ is related to $\boldsymbol{S}_{\text {int }}$ as

$$
\pi_{1} \boldsymbol{S}_{\mathrm{int}}=\mathcal{G} P_{0} \pi_{1} \boldsymbol{\Sigma}, \quad\langle S|=\left\langle\omega_{l}\right| \xi_{0} P_{0} \otimes P_{0} \pi_{1} \boldsymbol{\Sigma},
$$

\footnotetext{
${ }^{12}$ The associative product $m_{2}$ can, for example, be defined by using the Witten's star product as $m_{2}\left(\Phi_{1}, \Phi_{2}\right)=(-1)^{\operatorname{deg}\left(\Phi_{1}\right)} \Phi_{1} * \Phi_{2}$.
} 
and satisfies the Dyson-Schwinger equation

$$
\pi_{1} \boldsymbol{\Sigma}=\sum_{n=0}^{\infty} \pi_{1} \boldsymbol{A}_{n+2}\left(\left(P_{0} \pi_{1}-\Delta \pi_{1} \boldsymbol{\Sigma}\right)^{\otimes(n+2)}\right)
$$

where $\Delta=Q^{+} \mathcal{G}-\Xi \pi^{1}$. These basic relations and the differential equations (4.11) have the same form as those of the heterotic string field theory, and therefore it is easy to show that if we suppose that the two-parameter extension $\boldsymbol{\Sigma}(s, t)$ follows the extended Dyson-Schwinger equation

$$
\pi_{1} \boldsymbol{\Sigma}(s, t)=\sum_{n=0}^{\infty} \pi_{1} \boldsymbol{A}_{n+2}(s, t)\left(\left(P_{0} \pi_{1}-\Delta(s, t) \pi_{1} \boldsymbol{\Sigma}(s, t)\right)^{\otimes(n+2)}\right)
$$

with

$$
\Delta(s, t)=Q^{+} \mathcal{G}(s, t)-t \Xi \pi^{1}, \quad \mathcal{G}(s, t)=\pi^{0}+(t X+s) \pi^{1},
$$

$\boldsymbol{\Sigma}(s, t)$ commutes with $\boldsymbol{Q}$ and $\boldsymbol{\eta}$,

$$
[\boldsymbol{Q}, \boldsymbol{\Sigma}(s, t)]=0, \quad[\boldsymbol{\eta}, \boldsymbol{\Sigma}(s, t)]=0,
$$

and satisfies

$$
\partial_{t} \boldsymbol{\Sigma}(s, t)=[\boldsymbol{Q}, \boldsymbol{\rho}(s, t)], \quad \partial_{s} \boldsymbol{\Sigma}(s, t)=[\boldsymbol{\eta}, \boldsymbol{\rho}(s, t)],
$$

with certain $\boldsymbol{\rho}(s, t)$ determined in a manner similar to that for the heterotic string given in appendix D. The relations (4.27b) lead to the same equation as (2.71), from which we find that $\langle S(s, t)|=\left\langle\omega_{l}\right| \xi_{0} P_{0} \otimes P_{0} \pi_{1} \boldsymbol{\Sigma}(s, t)$ satisfies the equation

$$
\partial_{t}\langle S(s, t)|-\partial_{s}\langle S(s, t)| X_{0}=0,
$$

with

$$
\langle S(s, t)| X_{0}=\sum_{n=0}^{\infty}\left\langle S_{n+3}(s, t)\right|\left(X_{0} \otimes \mathbb{I}_{n+2}+\mathbb{I} \otimes \sum_{r=0}^{n+1} \mathbb{I}_{r} \otimes X_{0} \otimes \mathbb{I}_{n-r+1}\right),
$$

except for the terms which vanish when acting on physical states. Consequently, the physical S-matrix at the tree level can be rewritten as

$$
\left\langle S^{\text {phys }}\right|=\sum_{n=0}^{\infty} \sum_{p=0}^{n+1}\left\langle\left. S^{(0)}\right|^{2(n-p+1)}\left(X_{0}\right)^{p} \hat{\mathcal{P}},\right.
$$

and agrees with that in the first-quantized formulation since it has the form of integral of smooth functions (sections) with picture changed external states over the whole open-string moduli spaces.

\section{Summary and discussion}

In this paper, we have shown that the tree-level physical S-matrices derived from the homotopy-algebra-based superstring field theories reproduce those obtained in the firstquantized formulation. For the heterotic string field theory, the differential equations (2.21) 
for the (basic building blocks of) the string products $\boldsymbol{B}(s, t)$ play a key role. Utilizing these differential equations we can eventually derive a sequence of decent equations (2.82) for the amplitudes. They allow us to rewrite the physical S-matrix in such a way that the equivalence to the one obtained in the first-quantized formulation is transparent. The extension to the type II and open superstring field theories has been straightforward since the key differential equations have essentially the same form. The open superstring field theory considered in this paper is a generalization of the previously constructed one and provides various theories realizing different triangulations of the moduli space.

It is interesting to extend the proof to the S-matrix at the loop level. In order to consider it, however, we need to extend the discussion to the one based on the quantum (or loop) homotopy algebras [31, 48, 49]. For closed superstring field theories, it is related to the problem of how to avoid the unphysical spurious singularities [32, 50]. A consistent superstring field theory needs to be constructed to provide an algorithm that calculates the scattering amplitude as an integral over the moduli space that smoothly connects the contributions from various Feynman diagrams without hitting spurious singularities [51]. Some recent achievements [52-56] will help further progress.

There are many other interesting issues that can only be performed by means of the string field theories [57], and further studies are expected to be done.

\section{Acknowledgments}

The author would like to thank Tatsuya Sugimoto for collaboration in the early stage of this work. He also would like to thank Jakub Vošmera for drawing his attention to refs. [39, 40]. He also would like to express his appreciation to organizers of "Fundamental Aspects of String Theory" ICTP-SAIFR, Sao Paulo 1-12 June 2020, in particular Nathan Berkovits, for providing exciting atmosphere through the online workshop. This work was supported in part by JSPS Grant-in-Aid for Scientific Research (C) Grant Number JP18K03645.

\section{A S-matrix via HPT}

In this appendix, we derive the tree-level S-matrix of the heterotic string field theory (2.54) as the almost minimal model of the $L_{\infty}$ algebra $\left(\mathcal{H}^{\text {res }}, \Omega, \boldsymbol{L}\right)$ by means of HPT. ${ }^{13}$

Consider two chain complexes $\left(\mathcal{H}^{\text {res }}, d=Q\right)$ and $\left(\mathcal{H}_{0}, D=Q P_{0}\right)$ with chain maps

$$
p=P_{0}: \mathcal{H}^{\mathrm{res}} \rightarrow \mathcal{H}_{0}, \quad i=P_{0}: \mathcal{H}_{0} \hookrightarrow \mathcal{H}^{\mathrm{res}}
$$

satisfying

$$
p i=\mathbb{I}_{\mathcal{H}_{0}}=P_{0}, \quad i p=\mathbb{I}_{\mathcal{H}^{\text {res }}}+h d+d h=\mathbb{I}-Q^{+} Q-Q Q^{+} .
$$

The chain complex $\left(\mathcal{H}_{0}, Q P_{0}\right)$, with the gauge conditions, defines the relative BRST cohomology [59-61]. In order to obtain the S-matrix (2.54b) we first lift these equivalence data

\footnotetext{
${ }^{13}$ The minimal model usually considered $[46,47]$ is the S-matrix naturally appeared in the light-cone gauge, in which all the unphysical model is gauged away [58].
} 
to those acting on the corresponding symmetrized tensor algebra defined by eqs. (2.49): $\left(\mathcal{S H}^{\mathrm{res}}, \boldsymbol{d}=\boldsymbol{Q}\right)$ and $\left(\mathcal{S} \mathcal{H}_{0}, \boldsymbol{D}=\boldsymbol{Q P}\right)$ with

$$
\hat{\boldsymbol{p}}=\hat{\boldsymbol{P}}: \mathcal{S H}^{\mathrm{res}} \rightarrow \mathcal{S} \mathcal{H}_{0}, \quad \hat{\boldsymbol{i}}=\hat{\boldsymbol{P}}: \mathcal{S} \mathcal{H}_{0} \hookrightarrow \mathcal{S} \mathcal{H}^{\mathrm{res}}
$$

satisfying

$$
\hat{\boldsymbol{p}} \hat{\boldsymbol{i}}=\hat{\boldsymbol{P}}, \quad \hat{\boldsymbol{i}} \hat{\boldsymbol{p}}=\hat{\boldsymbol{I}}+\boldsymbol{H Q}+\boldsymbol{Q H} .
$$

Then, if we perturb $\boldsymbol{Q}$ by $\boldsymbol{L}_{\text {int }}$ so that $\left(\boldsymbol{Q}+\boldsymbol{L}_{\text {int }}\right)^{2}=0$, the homological perturbation lemma tells us that the equivalence data are deformed as

$$
\begin{aligned}
\boldsymbol{d}^{\prime} & =\boldsymbol{Q}+\boldsymbol{L}_{\mathrm{int}}=\boldsymbol{L} \\
\hat{\boldsymbol{i}}^{\prime} & =\left(\hat{\boldsymbol{I}}-\boldsymbol{H} \boldsymbol{L}_{\mathrm{int}}\right)^{-1} \hat{\boldsymbol{P}} \\
\hat{\boldsymbol{p}}^{\prime} & =\hat{\boldsymbol{P}}\left(\hat{\boldsymbol{I}}-\boldsymbol{L}_{\mathrm{int}} \boldsymbol{H}\right)^{-1} \\
\boldsymbol{D}^{\prime} & =\boldsymbol{Q P}+\hat{\boldsymbol{P}} \boldsymbol{L}_{\mathrm{int}}\left(\hat{\boldsymbol{I}}-\boldsymbol{H} \boldsymbol{L}_{\mathrm{int}}\right)^{-1} \hat{\boldsymbol{P}}=\boldsymbol{Q P}+\boldsymbol{S}_{\mathrm{int}}, \\
\boldsymbol{h}^{\prime} & =\boldsymbol{H}\left(\hat{\boldsymbol{I}}-\boldsymbol{L}_{\mathrm{int}} \boldsymbol{H}\right)^{-1}=\left(\hat{\boldsymbol{I}}-\boldsymbol{H} \boldsymbol{L}_{\mathrm{int}}^{-1}\right) \boldsymbol{H} .
\end{aligned}
$$

We confirm below that the almost minimal model $\left(\mathcal{S} \mathcal{H}_{0}, \boldsymbol{D}^{\prime}\right)$ is actually $L_{\infty}$-quasi-isomorphic to $\left(\mathcal{S} \mathcal{H}^{\text {res }}, \boldsymbol{d}^{\prime}=\boldsymbol{L}\right)$ by showing that the deformed maps $\hat{\boldsymbol{i}}^{\prime}$ and $\hat{\boldsymbol{p}}^{\prime}$ are cohomomorphisms, and $\boldsymbol{D}^{\prime}$ is a coderivation.

Note that coderivation $\boldsymbol{D}$ and cohomomorphism $\hat{\boldsymbol{F}}$ satisfy

$$
\begin{aligned}
\Delta \boldsymbol{D} & =(\boldsymbol{D} \otimes \mathbb{I}+\mathbb{I} \otimes \boldsymbol{D}) \Delta, \\
\Delta \hat{\boldsymbol{F}} & =(\hat{\boldsymbol{F}} \otimes \hat{\boldsymbol{F}}) \Delta,
\end{aligned}
$$

with respect to the coproduct $\Delta$. On the other hand, the homotopy operator $\boldsymbol{H}$ defined by $(2.49 \mathrm{a})$ itself does not have any good property under the coproduct $\Delta$. We have

$$
\begin{aligned}
\Delta \boldsymbol{H}= & \sum_{r, s, t, u=0}^{\infty}\left(\begin{array}{c}
r+t \\
r
\end{array}\right)\left(\begin{array}{c}
s+u \\
u
\end{array}\right) \\
& \times\left\{\left(-Q^{+}\right) \wedge \mathbb{I}^{\wedge r} \wedge P_{0}^{\wedge s} \otimes \mathbb{I}^{\wedge t} \wedge P_{0}^{\wedge u}+\mathbb{I}^{\wedge r} \wedge P_{0}^{\wedge s} \otimes\left(-Q^{+}\right) \wedge \mathbb{I}^{\wedge t} \wedge P_{0}^{\wedge u}\right\} \Delta .
\end{aligned}
$$

However, the complicated factor in front of the $\Delta$ in the right hand side becomes simple if it acts on $(\hat{\boldsymbol{P}} \otimes \boldsymbol{A}+\boldsymbol{A} \otimes \hat{\boldsymbol{P}})$ or $(\boldsymbol{H} \otimes \hat{\boldsymbol{I}}-\hat{\boldsymbol{I}} \otimes \boldsymbol{H})$ with any map $\boldsymbol{A}$ on $\mathcal{S} \mathcal{H}^{\text {res }}$, and we find that

$$
\begin{aligned}
& \sum_{r, s, t, u=0}^{\infty}\left(\begin{array}{c}
r+t \\
r
\end{array}\right)\left(\begin{array}{c}
s+u \\
u
\end{array}\right) \\
& \quad \times\left(\left(-Q^{+}\right) \wedge \mathbb{I}^{\wedge r} \wedge P_{0}^{\wedge s} \otimes \mathbb{I}^{\wedge t} \wedge P_{0}^{\wedge u}+\mathbb{I}^{\wedge r} \wedge P_{0}^{\wedge s} \otimes\left(-Q^{+}\right) \wedge \mathbb{I}^{\wedge t} \wedge P_{0}^{\wedge u}\right)(\hat{\boldsymbol{P}} \otimes \boldsymbol{A}+\boldsymbol{A} \otimes \hat{\boldsymbol{P}}) \\
& =\hat{\boldsymbol{P}} \otimes \boldsymbol{H} \boldsymbol{A}+\boldsymbol{H} \boldsymbol{A} \otimes \hat{\boldsymbol{P}},
\end{aligned}
$$


or

$$
\begin{aligned}
& \sum_{r, s, t, u=0}^{\infty}\left(\begin{array}{c}
r+t \\
r
\end{array}\right)\left(\begin{array}{c}
s+u \\
u
\end{array}\right) \\
& \quad \times\left(\left(-Q^{+}\right) \wedge \mathbb{I}^{\wedge r} \wedge P_{0}^{\wedge s} \otimes \mathbb{I}^{\wedge t} \wedge P_{0}^{\wedge u}+\mathbb{I}^{\wedge r} \wedge P_{0}^{\wedge s} \otimes\left(-Q^{+}\right) \wedge \mathbb{I}^{\wedge t} \wedge P_{0}^{\wedge u}\right)(\boldsymbol{H} \otimes \hat{\boldsymbol{I}}-\hat{\boldsymbol{I}} \otimes \boldsymbol{H}) \\
& =-\boldsymbol{H} \otimes \boldsymbol{H} .
\end{aligned}
$$

The first relation (A.13) is shown by using the formula

$$
\sum_{u=0}^{t}\left(\begin{array}{c}
r+t-u \\
r
\end{array}\right)\left(\begin{array}{c}
s+u \\
s
\end{array}\right)=\left(\begin{array}{c}
r+s+t+1 \\
t
\end{array}\right),
$$

which can be proven by mathematical induction with respect to $t$ as follows. For $t=0$ (and $t=1$ ), eq. (A.15) is clearly true. Suppose that it holds for $t=t_{0}$, then we have

$$
\begin{aligned}
\sum_{u=0}^{t_{0}+1}\left(\begin{array}{c}
r+t_{0}+1-u \\
r
\end{array}\right)\left(\begin{array}{c}
s+u \\
s
\end{array}\right)= & \left(\begin{array}{c}
r+t_{0}+1 \\
r
\end{array}\right)+\sum_{u=1}^{t_{0}}\left(\begin{array}{c}
r+t_{0}+1-u \\
r
\end{array}\right)\left(\begin{array}{c}
s+u \\
s
\end{array}\right)+\left(\begin{array}{c}
s+t_{0}+1 \\
s
\end{array}\right) \\
= & \sum_{u=0}^{t_{0}} \frac{r+t_{0}+1-u}{t_{0}+1}\left(\begin{array}{c}
r+t_{0}-u \\
r
\end{array}\right)\left(\begin{array}{c}
s+u \\
s
\end{array}\right) \\
& +\sum_{u=1}^{t_{0}+1} \frac{s+u}{t_{0}+1}\left(\begin{array}{c}
r+t_{0}+1-u \\
r
\end{array}\right)\left(\begin{array}{c}
s+u-1 \\
s
\end{array}\right) \\
= & \frac{r+s+t_{0}+2}{t_{0}+1} \sum_{u=0}^{t_{0}}\left(\begin{array}{c}
r+t_{0}-u \\
r
\end{array}\right)\left(\begin{array}{c}
s+u \\
s
\end{array}\right) \\
= & \left(\begin{array}{c}
r+s+t_{0}+2 \\
t_{0}+1
\end{array}\right),
\end{aligned}
$$

using the assumption of the induction in the last equality. We used simple relations of the binomial coefficients

$$
t\left(\begin{array}{c}
t-1 \\
r
\end{array}\right)=(t-r)\left(\begin{array}{l}
t \\
r
\end{array}\right)=(r+1)\left(\begin{array}{c}
t \\
r+1
\end{array}\right),
$$

for $0 \leq r<t \in \mathbb{Z}$, and the relation

$$
\begin{aligned}
\left(\begin{array}{c}
r+t_{0}+1-u \\
r
\end{array}\right)\left(\begin{array}{c}
s+u \\
s
\end{array}\right)= & \frac{r+t_{0}+1-u}{t_{0}+1}\left(\begin{array}{c}
r+t_{0}-u \\
r
\end{array}\right)\left(\begin{array}{c}
s+u \\
s
\end{array}\right) \\
& +\frac{s+u}{t_{0}+1}\left(\begin{array}{c}
r+t_{0}+1-u \\
r
\end{array}\right)\left(\begin{array}{c}
s+u-1 \\
s
\end{array}\right),
\end{aligned}
$$

for $1 \leq u \leq t_{0}$, which can easily be confirmed using (A.17). Since eq. (A.16) shows that eq. (A.15) is true for $t=t_{0}+1$, eq. (A.15) is true by mathematical induction.

The second relation (A.14) follows from the relation

$$
\frac{1}{t !}\left(\mathbb{I}^{\wedge t} \wedge P_{0}^{\wedge u}\right) \frac{1}{(t+u+1) !} \sum_{\alpha=0}^{t+u}\left(\mathbb{I}^{\wedge(t+u-\alpha)} \wedge P_{0}^{\wedge \alpha}\right)=\frac{1}{(t+1) !} \sum_{\alpha=0}^{t}\left(\mathbb{I}^{\wedge(t-\alpha)} \wedge P_{0}^{\wedge(u+\alpha)}\right),
$$


and the formula

$$
\sum_{\alpha=0}^{u}\left(\begin{array}{c}
r+t-s-\alpha+1 \\
r-s
\end{array}\right)\left(\begin{array}{c}
s+\alpha \\
s
\end{array}\right)+\sum_{\alpha=0}^{s}\left(\begin{array}{c}
r+t-u-\alpha+1 \\
t-u
\end{array}\right)\left(\begin{array}{c}
u+\alpha \\
u
\end{array}\right)=\left(\begin{array}{c}
r+t+2 \\
r+1
\end{array}\right)
$$

for $r \geq s$ and $t \geq u$. In order to show the relation (A.19), we first prove the formula

$$
\sum_{\beta=0}^{u}\left(\begin{array}{l}
u \\
\beta
\end{array}\right) \frac{(t+u-\alpha-\beta) !}{(t-\alpha) !} \frac{(\alpha+\beta) !}{\alpha !}=\frac{(t+u+1) !}{(t+1) !},
$$

by mathematical induction with respect to $u$ as follows. For $u=0$, it clearly holds. Suppose that it holds for $u=u_{0}$, then

$$
\begin{aligned}
& \sum_{\beta=0}^{u_{0}+1}\left(\begin{array}{c}
u_{0}+1 \\
\beta
\end{array}\right) \frac{\left(t+u_{o}+1-\alpha-\beta\right) !}{(t-\alpha) !} \frac{(\alpha+\beta) !}{\alpha !} \\
& =\frac{\left(t+u_{0}+1-\alpha\right) !}{(t-\alpha) !}+\sum_{\beta=1}^{u_{0}}\left(\begin{array}{c}
u_{0}+1 \\
\beta
\end{array}\right) \frac{\left(t+u_{0}+1-\alpha-\beta\right) !}{(t-\alpha) !} \frac{(\alpha+\beta) !}{\alpha !}+\frac{\left(\alpha+u_{0}+1\right) !}{\alpha !} \\
& =\sum_{\beta=0}^{u_{0}}\left(\begin{array}{c}
u_{0} \\
\beta
\end{array}\right) \frac{\left(t+u_{0}+1-\alpha-\beta\right)}{(t-\alpha) !} \frac{(\alpha+\beta) !}{\alpha !}+\sum_{\beta=1}^{u_{0}+1}\left(\begin{array}{c}
u_{0} \\
\beta-1
\end{array}\right) \frac{\left(t+u_{0}+1-\alpha-\beta\right) !}{(t-\alpha) !} \frac{(\alpha+\beta) !}{\alpha !} \\
& =\left(t+u_{0}+2\right) \sum_{\beta=0}^{u_{0}}\left(\begin{array}{c}
u_{0} \\
\beta
\end{array}\right) \frac{\left(t+u_{0}-\alpha-\beta\right) !}{(t-\alpha) !} \frac{(\alpha+\beta) !}{\alpha !} \\
& =\frac{\left(t+u_{0}+2\right) !}{(t+1) !},
\end{aligned}
$$

by using the relation

$$
\left(\begin{array}{c}
u_{0}+1 \\
\beta
\end{array}\right)=\left(\begin{array}{c}
u_{0} \\
\beta
\end{array}\right)+\left(\begin{array}{c}
u_{0} \\
\beta-1
\end{array}\right)
$$

for $1 \leq \beta \leq u_{0}$. This shows that (A.21) holds for $u=u_{0}+1$, and hence the formula (A.21) is proven by mathematical induction. Using this formula (A.21), the left hand side of the relation (A.19) can be calculated by noting that $\frac{1}{t !} \mathbb{I}^{\wedge t}$ is the identity on $\left(\mathcal{H}^{\text {res }}\right)^{\wedge t}$ :

$$
\begin{aligned}
& \frac{1}{t !}\left(\mathbb{I}^{\wedge t} \wedge P_{0}^{\wedge u}\right) \frac{1}{(t+u+1) !} \sum_{\alpha=0}^{t+u}\left(\mathbb{I}^{\wedge(t+u-\alpha)} \wedge P_{0}^{\wedge \alpha}\right) \\
& =\frac{1}{(t+u+1) !} \sum_{\beta=0}^{u} \sum_{\alpha=\beta}^{t+\beta}\left(\begin{array}{l}
u \\
\beta
\end{array}\right) \frac{(t+u-\alpha) !}{(t+\beta-\alpha) !} \frac{\alpha !}{(\alpha-\beta) !} \mathbb{I}^{\wedge(t+\beta-\alpha)} \wedge P_{0}^{\wedge(u+\alpha-\beta)} \\
& =\frac{1}{(t+u+1) !} \sum_{\alpha=0}^{t}\left(\sum_{\beta=0}^{u}\left(\begin{array}{l}
u \\
\beta
\end{array}\right) \frac{(t+u-\alpha-\beta) !}{(t-\alpha)} \frac{(\alpha+\beta) !}{\alpha !}\right) \mathbb{I}^{\wedge(t-\alpha)} \wedge P_{0}^{\wedge(u+\alpha)} \\
& =\frac{1}{(t+1) !} \sum_{\alpha=0}^{t} \mathbb{I}^{\wedge(t-\alpha)} \wedge P_{0}^{\wedge(u+\alpha)} .
\end{aligned}
$$


The proof of the formula (A.20) is the following. We assume that $s \geq u$, which is possible without loss of generality since the formula is symmetric with respect to $s$ and $u$, and use the mathematical induction with respect to $u$. First, rewrite the formula as

$$
\sum_{\alpha=0}^{u}\left(\begin{array}{c}
r+t-s-\alpha+1 \\
r-s
\end{array}\right)\left(\begin{array}{c}
s+\alpha \\
s
\end{array}\right)+\sum_{\alpha=0}^{s}\left(\begin{array}{c}
r+t-s-u+\alpha+1 \\
r-s+\alpha+1
\end{array}\right)\left(\begin{array}{c}
s+u-\alpha \\
s-\alpha
\end{array}\right)=\left(\begin{array}{c}
r+t+2 \\
r+1
\end{array}\right) .
$$

For $u=0$, the left hand side is calculated as

$$
\begin{aligned}
& \left(\begin{array}{c}
r+t-s+1 \\
r-s
\end{array}\right)+\sum_{\alpha=0}^{s}\left(\begin{array}{c}
r+t-s+\alpha+1 \\
r-s+\alpha+1
\end{array}\right) \\
& =\left(\begin{array}{c}
r+t-s+1 \\
r-s
\end{array}\right)+\left(\begin{array}{c}
r+t-s+1 \\
r-s+1
\end{array}\right)+\sum_{\alpha=1}^{s}\left(\begin{array}{c}
r+t-s+\alpha+1 \\
r-s+\alpha+1
\end{array}\right) \\
& =\cdots \\
& =\left(\begin{array}{c}
r+t-s+k+1 \\
r-s+k
\end{array}\right)+\left(\begin{array}{c}
r+t-s+k+1 \\
r-s+k+1
\end{array}\right)+\sum_{\alpha=k+1}^{s}\left(\begin{array}{c}
r+t-s+\alpha+1 \\
r-s+\alpha+1
\end{array}\right) \\
& \left.=\cdots \begin{array}{c}
r+t+1 \\
r
\end{array}\right)+\left(\begin{array}{c}
r+t+1 \\
r+1
\end{array}\right)=\left(\begin{array}{c}
r+t+2 \\
r+1
\end{array}\right),
\end{aligned}
$$

by using the relation (A.23) repeatedly. Then, suppose that it is true for $u=u_{0}$. The left hand side of (A.25) for $u=u_{0}+1$ can be calculated as

$$
\begin{aligned}
\sum_{\alpha=0}^{u_{0}+1} & \left(\begin{array}{c}
r+t-s-\alpha+1 \\
r-s
\end{array}\right)\left(\begin{array}{c}
s+\alpha \\
s
\end{array}\right)+\sum_{\alpha=0}^{s}\left(\begin{array}{c}
r+t-s-u_{0}+\alpha \\
r-s+\alpha+1
\end{array}\right)\left(\begin{array}{c}
s+u_{0}-\alpha+1 \\
s-\alpha
\end{array}\right) \\
= & \sum_{\alpha=0}^{u_{0}}\left(\begin{array}{c}
r+t-s-\alpha+1 \\
r-s
\end{array}\right)\left(\begin{array}{c}
s+\alpha \\
s
\end{array}\right)+\left(\begin{array}{c}
r+t-s-u_{0} \\
r-s
\end{array}\right)\left(\begin{array}{c}
s+u_{0}+1 \\
s
\end{array}\right) \\
& +\left(\begin{array}{c}
r+t-s-u_{0} \\
r-s+1
\end{array}\right)\left(\begin{array}{c}
s+u_{0}+1 \\
s
\end{array}\right)+\sum_{\alpha=1}^{s}\left(\begin{array}{c}
r+t-s-u_{0}+\alpha \\
r-s+\alpha+1
\end{array}\right)\left(\begin{array}{c}
s+u_{0}-\alpha+1 \\
s-\alpha
\end{array}\right) \\
= & \sum_{\alpha=0}^{u_{0}}\left(\begin{array}{c}
r+t-s-\alpha+1 \\
r-s
\end{array}\right)\left(\begin{array}{c}
s+\alpha \\
s
\end{array}\right) \\
& +\left(\begin{array}{c}
r+t-s-u_{0}+1 \\
r-s+1
\end{array}\right)\left(\begin{array}{c}
s+u_{0}+1 \\
s
\end{array}\right)+\sum_{\alpha=1}^{s}\left(\begin{array}{c}
r+t-s-u_{0}+\alpha \\
r-s+\alpha+1
\end{array}\right)\left(\begin{array}{c}
s+u_{0}-\alpha+1 \\
s-\alpha
\end{array}\right) \\
= & \sum_{\alpha=0}^{u_{0}}\left(\begin{array}{c}
r+t-s-\alpha+1 \\
r-s
\end{array}\right)\left(\begin{array}{c}
s+\alpha \\
s
\end{array}\right) \\
& +\left(\begin{array}{c}
r+t-s-u_{0}+1 \\
r-s+1
\end{array}\right)\left(\begin{array}{c}
s+u_{0} \\
s
\end{array}\right)+\left(\begin{array}{c}
r+t-s-u_{0}+1 \\
r-s+1
\end{array}\right)\left(\begin{array}{c}
s+u_{0} \\
s-1
\end{array}\right) \\
& +\left(\begin{array}{c}
r+t-s-u_{0}+1 \\
r-s+2
\end{array}\right)\left(\begin{array}{c}
s+u_{0} \\
s-1
\end{array}\right)+\sum_{\alpha=2}^{s}\left(\begin{array}{c}
r+t-s-u_{0}+\alpha \\
r-s+\alpha+1
\end{array}\right)\left(\begin{array}{c}
s+u_{0}-\alpha+1 \\
s-\alpha
\end{array}\right)
\end{aligned}
$$




$$
\begin{aligned}
& =\sum_{\alpha=0}^{u_{0}}\left(\begin{array}{c}
r+t-s-\alpha+1 \\
r-s
\end{array}\right)\left(\begin{array}{c}
s+\alpha \\
s
\end{array}\right)+\left(\begin{array}{c}
r+t-s-u_{0}+1 \\
r-s+1
\end{array}\right)\left(\begin{array}{c}
s+u_{0} \\
s
\end{array}\right) \\
& +\left(\begin{array}{c}
r+t-s-u_{0}+2 \\
r-s+2
\end{array}\right)\left(\begin{array}{c}
s+u_{0} \\
s-1
\end{array}\right) \\
& +\sum_{\alpha=2}^{s}\left(\begin{array}{c}
r+t-s-u_{0}+\alpha \\
r-s+\alpha+1
\end{array}\right)\left(\begin{array}{c}
s+u_{0}-\alpha+1 \\
s-\alpha
\end{array}\right) \\
& =\sum_{\alpha=0}^{u_{0}}\left(\begin{array}{c}
r+t-s-\alpha+1 \\
r-s
\end{array}\right)\left(\begin{array}{c}
s+\alpha \\
s
\end{array}\right)+\left(\begin{array}{c}
r+t-s-u_{0}+1 \\
r-s+1
\end{array}\right)\left(\begin{array}{c}
s+u_{0} \\
s
\end{array}\right) \\
& +\left(\begin{array}{c}
r+t-s-u_{0}+2 \\
r-s+2
\end{array}\right)\left(\begin{array}{c}
s+u_{0}-1 \\
s-1
\end{array}\right)+\left(\begin{array}{c}
r+t-s-u_{0}+2 \\
r-s+2
\end{array}\right)\left(\begin{array}{c}
s+u_{0}-1 \\
s-2
\end{array}\right) \\
& +\left(\begin{array}{c}
r+t-s-u_{0}+2 \\
r-s+3
\end{array}\right)\left(\begin{array}{c}
s+u_{0}-1 \\
s-2
\end{array}\right)+\sum_{\alpha=3}^{s}\left(\begin{array}{c}
r+t-s-u_{0}+\alpha \\
r-s+\alpha+1
\end{array}\right)\left(\begin{array}{c}
s+u_{0}-\alpha+1 \\
s-\alpha
\end{array}\right) \\
& =\cdots \\
& =\sum_{\alpha=0}^{u_{0}}\left(\begin{array}{c}
r+t-s-\alpha+1 \\
r-s
\end{array}\right)\left(\begin{array}{c}
s+\alpha \\
s
\end{array}\right)+\sum_{\alpha=0}^{k-1}\left(\begin{array}{c}
r+t-s-u_{0}+\alpha+1 \\
r-s+\alpha+1
\end{array}\right)\left(\begin{array}{c}
s+u_{0}-\alpha \\
s-\alpha
\end{array}\right) \\
& +\left(\begin{array}{c}
r+t-s-u_{0}+k \\
r-s+k
\end{array}\right)\left(\begin{array}{c}
s+u_{0}-k+2 \\
s-k+1
\end{array}\right) \\
& +\sum_{\alpha=k}^{s}\left(\begin{array}{c}
r+t-s-u_{0}+\alpha \\
r-s+\alpha+1
\end{array}\right)\left(\begin{array}{c}
s+u_{0}-\alpha+1 \\
s-\alpha
\end{array}\right) \\
& =\cdots \\
& =\sum_{\alpha=0}^{u_{0}}\left(\begin{array}{c}
r+t-s-\alpha+1 \\
r-s
\end{array}\right)\left(\begin{array}{c}
s+\alpha \\
s
\end{array}\right)+\sum_{\alpha=0}^{s-1}\left(\begin{array}{c}
r+t-s-u_{0}+\alpha+1 \\
r-s+\alpha+1
\end{array}\right)\left(\begin{array}{c}
s+u_{0}-\alpha \\
s-\alpha
\end{array}\right) \\
& +\left(\begin{array}{c}
r+t-u_{0} \\
r
\end{array}\right)+\left(\begin{array}{c}
r+t-u_{0} \\
r+1
\end{array}\right) \\
& =\sum_{\alpha=0}^{u_{0}}\left(\begin{array}{c}
r+t-s-\alpha+1 \\
r-s
\end{array}\right)\left(\begin{array}{c}
s+\alpha \\
s
\end{array}\right)+\sum_{\alpha=0}^{s}\left(\begin{array}{c}
r+t-s-u_{0}+\alpha+1 \\
r-s+\alpha+1
\end{array}\right)\left(\begin{array}{c}
s+u_{0}-\alpha \\
s-\alpha
\end{array}\right) \\
& =\left(\begin{array}{c}
r+t+2 \\
r+1
\end{array}\right) \text {. }
\end{aligned}
$$

The last equality follows from the assumption of the induction. Hence, the formula (A.20) is true for ${ }^{\forall} u$ by mathematical induction.

Using these properties of $\boldsymbol{H}$, we can show, order by order in the power of $\boldsymbol{H}$, that the linear map on $\mathcal{S H}_{0}$,

$$
\hat{\boldsymbol{i}}^{\prime}=\left(\hat{\boldsymbol{I}}-\boldsymbol{H} \boldsymbol{L}_{\mathrm{int}}\right)^{-1} \hat{\boldsymbol{P}}=\sum_{n=0}^{\infty}\left(\boldsymbol{H} \boldsymbol{L}_{\mathrm{int}}\right)^{n} \hat{\boldsymbol{P}}
$$


satisfies the property of the cohomomorphism

$$
\Delta \hat{\boldsymbol{i}}^{\prime}=\left(\hat{\boldsymbol{i}}^{\prime} \otimes \hat{\boldsymbol{i}}^{\prime}\right) \Delta .
$$

At $\mathcal{O}\left((\mathbf{H})^{\mathbf{0}}\right)$, eq. (A.29) reduces to $\Delta \hat{\boldsymbol{P}}=(\hat{\boldsymbol{P}} \otimes \hat{\boldsymbol{P}}) \Delta$, which follows from the definition $(2.49 \mathrm{~b})$ of $\hat{\boldsymbol{P}}$. For $\mathcal{O}\left((\boldsymbol{H})^{n}\right)$ with $n \geq 1$, we use the mathematical induction. If we note that

$$
\Delta \boldsymbol{L}_{\mathrm{int}} \hat{\boldsymbol{P}}=\left(\hat{\boldsymbol{P}} \otimes \boldsymbol{L}_{\mathrm{int}} \hat{\boldsymbol{P}}+\boldsymbol{L}_{\mathrm{int}} \hat{\boldsymbol{P}} \otimes \hat{\boldsymbol{P}}\right) \Delta,
$$

and the factor in the right hand side has the form mentioned above, we can see that $\mathcal{O}(\boldsymbol{H})$ of eq. (A.29),

$$
\Delta\left(\boldsymbol{H} \boldsymbol{L}_{\mathrm{int}} \hat{\boldsymbol{P}}\right)=\left(\hat{\boldsymbol{P}} \otimes \boldsymbol{H} \boldsymbol{L}_{\mathrm{int}} \hat{\boldsymbol{P}}+\boldsymbol{H} \boldsymbol{L}_{\mathrm{int}} \hat{\boldsymbol{P}} \otimes \hat{\boldsymbol{P}}\right) \Delta,
$$

also holds thanks to the relation (A.13). If we suppose that $\mathcal{O}\left((\boldsymbol{H})^{n_{0}}\right)$ of (A.29),

$$
\Delta\left(\left(\boldsymbol{H} \boldsymbol{L}_{\mathrm{int}}\right)^{n_{0}} \hat{\boldsymbol{P}}\right)=\left(\sum_{m=0}^{n_{0}}\left(\left(\boldsymbol{H} \boldsymbol{L}_{\mathrm{int}}\right)^{n_{0}-m} \hat{\boldsymbol{P}} \otimes\left(\boldsymbol{H} \boldsymbol{L}_{\mathrm{int}}\right)^{m} \hat{\boldsymbol{P}}\right)\right) \Delta
$$

holds, then from

$$
\begin{aligned}
\Delta \boldsymbol{L}_{\mathrm{int}}\left(\boldsymbol{H} \boldsymbol{L}_{\mathrm{int}}\right)^{n_{0}} \hat{\boldsymbol{P}} \\
=\left(\hat{\boldsymbol{P}} \otimes \boldsymbol{L}_{\mathrm{int}}\left(\boldsymbol{H} \boldsymbol{L}_{\mathrm{int}}\right)^{n_{0}} \hat{\boldsymbol{P}}+\boldsymbol{L}_{\mathrm{int}}\left(\boldsymbol{H} \boldsymbol{L}_{\mathrm{int}}\right)^{n_{0}} \hat{\boldsymbol{P}} \otimes \hat{\boldsymbol{P}}\right. \\
\left.\quad+(\boldsymbol{H} \otimes \hat{\boldsymbol{I}}-\hat{\boldsymbol{I}} \otimes \boldsymbol{H}) \sum_{m=0}^{n_{0}-1}\left(\boldsymbol{L}_{\mathrm{int}}\left(\boldsymbol{H} \boldsymbol{L}_{\mathrm{int}}\right)^{n_{0}-m-1} \hat{\boldsymbol{P}} \otimes \boldsymbol{L}_{\mathrm{int}}\left(\boldsymbol{H} \boldsymbol{L}_{\mathrm{int}}\right)^{m} \hat{\boldsymbol{P}}\right)\right) \Delta,
\end{aligned}
$$

the relation at $\mathcal{O}\left((\boldsymbol{H})^{n_{0}+1}\right)$,

$$
\Delta\left(\left(\boldsymbol{H} \boldsymbol{L}_{\mathrm{int}}\right)^{n_{0}+1} \hat{\boldsymbol{P}}\right)=\left(\sum_{m=0}^{n_{0}+1}\left(\left(\boldsymbol{H} \boldsymbol{L}_{\mathrm{int}}\right)^{n_{0}-m+1} \hat{\boldsymbol{P}} \otimes\left(\boldsymbol{H} \boldsymbol{L}_{\mathrm{int}}\right)^{m} \hat{\boldsymbol{P}}\right)\right) \Delta,
$$

holds again thanks to the relations (A.13) and (A.14). Hence, eq. (A.29) holds at any order, $\mathcal{O}\left((\boldsymbol{H})^{n}\right)$ with ${ }^{\forall} n$, due to the mathematical induction, and thus, the map $\hat{\boldsymbol{i}}^{\prime}$ is a cohomomorphism. Note that the cohomomorphism $\hat{\boldsymbol{i}}^{\prime}$ is cyclic with respect to $\Omega$ in the sense that it satisfies

$$
\langle\Omega| \pi_{1} \hat{\boldsymbol{i}}^{\prime} \otimes \pi_{1} \hat{\boldsymbol{i}}^{\prime}=\langle\Omega| P_{0} \otimes P_{0} .
$$

This follows from eqs. (2.42b), and the fact that the symplectic form $\langle\Omega|$ compatible with the decomposition (2.43) satisfies

$$
\langle\Omega| \mathbb{I} \otimes Q^{+}=\langle\Omega| Q^{+} \otimes \mathbb{I}, \quad\langle\Omega| \mathbb{I} \otimes P_{0}=\langle\Omega| P_{0} \otimes \mathbb{I} .
$$

We can also show that $\hat{\boldsymbol{p}}^{\prime}$ is a cohomomorphism using the similar relations to (A.13) and (A.14),

$$
\begin{gathered}
(\hat{\boldsymbol{P}} \otimes \boldsymbol{A}+\boldsymbol{A} \otimes \hat{\boldsymbol{P}}) \sum_{r, s, t, u=0}^{\infty}\left(\begin{array}{c}
r+t \\
r
\end{array}\right)\left(\begin{array}{c}
s+u \\
u
\end{array}\right) \\
\times\left(\left(-Q^{+}\right) \wedge \mathbb{I}^{\wedge r} \wedge P_{0}^{\wedge s} \otimes \mathbb{I}^{\wedge t} \wedge P_{0}^{\wedge u}+\mathbb{I}^{\wedge r} \wedge P_{0}^{\wedge s} \otimes\left(-Q^{+}\right) \wedge \mathbb{I}^{\wedge t} \wedge P_{0}^{\wedge u}\right) \\
=\hat{\boldsymbol{P}} \otimes \boldsymbol{A} \boldsymbol{H}+\boldsymbol{A} \boldsymbol{H} \otimes \hat{\boldsymbol{P}},
\end{gathered}
$$


and

$$
\begin{aligned}
(\boldsymbol{H} \otimes \hat{\boldsymbol{I}}-\hat{\boldsymbol{I}} \otimes \boldsymbol{H}) & \sum_{r, s, t, u=0}^{\infty}\left(\begin{array}{c}
r+t \\
r
\end{array}\right)\left(\begin{array}{c}
s+u \\
u
\end{array}\right) \\
& \times\left(\left(-Q^{+}\right) \wedge \mathbb{I}^{\wedge r} \wedge P_{0}^{\wedge s} \otimes \mathbb{I}^{\wedge t} \wedge P_{0}^{\wedge u}+\mathbb{I}^{\wedge r} \wedge P_{0}^{\wedge s} \otimes\left(-Q^{+}\right) \wedge \mathbb{I}^{\wedge t} \wedge P_{0}^{\wedge u}\right) \\
& =\boldsymbol{H} \otimes \boldsymbol{H},
\end{aligned}
$$

respectively, obtained by reversing the order of the factors. We omit the explicit proof since it is parallel to that for $\hat{\boldsymbol{i}}^{\prime}$.

\section{B Derivation of (2.54a)}

Note that the action (2.35) can also be written as a WZW-like form

$$
I[\Phi]=\int_{0}^{1} d t \Omega\left(\partial_{t} \Phi(t), \pi_{1} \boldsymbol{L}\left(e^{\wedge \Phi(t)}\right)\right),
$$

where $\Phi(t)$ is a one-parameter, $t \in[0,1]$, extension of the string field $\Phi$ satisfying $\Phi(1)=\Phi$ and $\Phi(0)=0$. This can easily be seen as follows. First, note that the action (B.1) agrees with the action (2.35) if we take $\Phi(t)=t \Phi$. On the other hand, it is easy to show that an arbitrary variation of the integrand becomes total $t$-derivative,

$$
\delta \Omega\left(\partial_{t} \Phi(t), \pi_{1} \boldsymbol{L}\left(e^{\wedge \Phi(t)}\right)\right)=\partial_{t} \Omega\left(\delta \Phi(t), \pi_{1} \boldsymbol{L}\left(e^{\wedge \Phi(t)}\right)\right) .
$$

Thus, the action (2.35) is not changed by deforming the $t$-dependence of $\Phi(t)$ as long as keeping the boundary condition: $\delta \Phi(0)=\delta \Phi(1)=0$. Then, we extend the classical solution (2.52) as

$$
\phi_{\mathrm{cl}}(t)=\pi_{1}\left(\hat{\boldsymbol{I}}-\boldsymbol{H} \boldsymbol{L}_{\mathrm{int}}\right)^{-1} \hat{\boldsymbol{P}}\left(e^{\wedge t \phi_{0}}\right)
$$

and evaluate the action as

$$
\begin{aligned}
I\left[\phi_{\mathrm{cl}}\right] & =\int_{0}^{1} d t \Omega\left(\pi_{1}\left(\hat{\boldsymbol{I}}-\boldsymbol{H} \boldsymbol{L}_{\mathrm{int}}\right)^{-1} \hat{\boldsymbol{P}}\left(\phi_{0} \wedge e^{\wedge t \phi_{0}}\right), \pi_{1} \boldsymbol{L}\left(\hat{\boldsymbol{I}}-\boldsymbol{H} \boldsymbol{L}_{\mathrm{int}}\right)^{-1} \hat{\boldsymbol{P}}\left(e^{\wedge t \phi_{0}}\right)\right) \\
& =\int_{0}^{1} d t\langle\Omega| \phi_{0} \otimes \pi_{1} \boldsymbol{S}\left(e^{\wedge t \phi_{0}}\right)
\end{aligned}
$$

using the bilinear map representation and the relation

$$
\left(\hat{\boldsymbol{I}}-\boldsymbol{H} \boldsymbol{L}_{\mathrm{int}}\right) \boldsymbol{L}\left(\hat{\boldsymbol{I}}-\boldsymbol{H} \boldsymbol{L}_{\mathrm{int}}\right)^{-1}=\boldsymbol{Q}+\hat{\boldsymbol{P}} \boldsymbol{L}_{\mathrm{int}}\left(\hat{\boldsymbol{I}}-\boldsymbol{H} \boldsymbol{L}_{\mathrm{int}}\right),
$$

that follows from (2.50a), (A.35), and $\boldsymbol{L}^{2}=\left(\boldsymbol{Q}+\boldsymbol{L}_{\text {int }}\right)^{2}=0$. The S-matrix generating functional $(2.54 \mathrm{a})$ is obtained by explicitly carrying out the $t$-integration. 


\section{Proof of (2.69)}

In order to explicitly investigate $\boldsymbol{\Sigma}(s, t)$, it is necessary to expand it in the number of inputs as (2.75a). The generalized Dyson-Schwinger equation (2.67) is then written as

$$
\begin{aligned}
\pi_{1} \boldsymbol{\Sigma}_{n+2}(s, t) & =\sum_{m=0}^{\infty} \pi_{1} \boldsymbol{B}_{m+2}(s, t)\left(\frac{1}{(m+2) !}\left(P_{0} \pi_{1}-\Delta(s, t) \sum_{l=0}^{n-m-1} \pi_{1} \boldsymbol{\Sigma}_{l+2}(s, t)\right)^{\wedge(m+2)}\right) \pi_{n+2}, \\
& \equiv \sum_{m=0}^{n} \pi_{1} \boldsymbol{B}_{m+2}(s, t)\left(D_{m+2}(s, t)\right) \pi_{n+2},
\end{aligned}
$$

where we defined

$$
D_{M}(s, t) \equiv \frac{1}{M !}\left(P_{0} \pi_{1}-\Delta(s, t) \pi_{1} \boldsymbol{\Sigma}(s, t)\right)^{\wedge M},
$$

for notational simplicity. Note that eq. (C.1) is a recurrence relation whose right hand side includes only $\boldsymbol{\Sigma}_{l+2}(s, t)$ with $l<n$. It determines all the $\boldsymbol{\Sigma}_{n+2}(s, t)(n \geq 0)$ recursively from the relation for $n=0: \pi_{1} \boldsymbol{\Sigma}_{2}(s, t)=\pi_{1} \boldsymbol{B}_{2}(s, t) P_{2}$. Then, eq. (2.69) can be proved by complete induction with respect to the number of external strings. For $n=0$, we have

$$
\begin{aligned}
{\left[\boldsymbol{Q}, \pi_{1} \boldsymbol{\Sigma}_{2}(s, t)\right] } & =\left[\boldsymbol{Q}, \pi_{1} \boldsymbol{B}_{2}(s, t) P_{2}\right]=0 \\
{\left[\boldsymbol{\eta}, \pi_{1} \boldsymbol{\Sigma}_{2}(s, t)\right] } & =\left[\boldsymbol{\eta}, \pi_{1} \boldsymbol{B}_{2}(s, t) P_{2}\right]=0,
\end{aligned}
$$

which follow from the relations for $\boldsymbol{B}_{2}(s, t)$ in eqs. (2.34). Next, suppose that

$$
\left[\boldsymbol{Q}, \pi_{1} \boldsymbol{\Sigma}_{l+2}(s, t)\right]=\left[\boldsymbol{\eta}, \pi_{1} \boldsymbol{\Sigma}_{l+2}(s, t)\right]=0
$$

for $0 \leq l \leq n-1$. Then, we find from eq. (C.1) that

$$
\begin{aligned}
& {\left[\boldsymbol{Q}, \pi_{1} \boldsymbol{\Sigma}_{n+2}(s, t)\right]} \\
& =-\sum_{l=0}^{n-1} \pi_{1} \boldsymbol{B}_{l+2}(s, t)\left(D_{l+1}(s, t) \wedge \pi(s) \pi_{1} \sum_{m=0}^{n-l-1} \boldsymbol{B}_{m+2}(s, t)\left(D_{m+2}(s, t)\right)\right) \pi_{n+2} \\
& \quad+\sum_{m=0}^{n-1} \pi_{1} \boldsymbol{B}_{m+2}(s, t)\left(D_{m+1}(s, t) \wedge \pi(s) \pi_{1} \boldsymbol{\Sigma}(s, t)\right) \pi_{n+2} \\
& =0,
\end{aligned}
$$

using the relation (2.34a) and

$$
[Q, \Delta(s, t)]=\pi(s)-P_{0} \mathcal{G}(s, t) .
$$

It was also used the fact that the internal states are generically off-shell. Hence, from the principle of mathematical induction, we can conclude that $\left[\boldsymbol{Q}, \pi_{1} \boldsymbol{\Sigma}(s, t)\right]=0$. 
Similarly, we find from eq. (C.1) that

$$
\begin{aligned}
& {\left[\boldsymbol{\eta}, \pi_{1} \boldsymbol{\Sigma}_{n+2}(s, t)\right]} \\
& =-\sum_{l=0}^{n-1} \pi_{1} \boldsymbol{B}_{l+2}(s, t)\left(D_{l+1}(s, t) \wedge t \pi_{1}^{1} \sum_{m=0}^{n-l-1} \boldsymbol{B}_{m+2}(s, t)\left(D_{m+2}(s, t)\right)\right) \pi_{n+2} \\
& \quad+\sum_{m=0}^{n-1} \pi_{1} \boldsymbol{B}_{m+2}(s, t)\left(D_{m+1}(s, t) \wedge t \pi_{1}^{1} \boldsymbol{\Sigma}(s, t)\right) \pi_{n+2} \\
& =0
\end{aligned}
$$

using the relation $(2.34 \mathrm{~b})$ and

$$
[\eta, \Delta(s, t)]=-t \pi^{1}
$$

with the off-shell-ness of the internal states. Hence, it can also be concluded that $\left[\boldsymbol{\eta}, \pi_{1} \boldsymbol{\Sigma}(s, t)\right]=0$ from the principle of mathematical induction.

\section{Proof of (2.70)}

The proof of (2.70) is given by complete induction with respect to the number of inputs. For $\boldsymbol{\Sigma}_{2}(s, t)=\boldsymbol{B}_{2}(s, t) P_{2}$, we have

$$
\pi_{1} \partial_{t} \boldsymbol{\Sigma}_{2}(s, t)=\left[\boldsymbol{Q}, \pi_{1} \boldsymbol{\rho}_{2}(s, t)\right],
$$

with $\pi_{1} \boldsymbol{\rho}_{2}(s, t)=\pi_{1} \boldsymbol{\lambda}_{2}(s, t) P_{2}$ using (2.33a) with $n=0$ :

$$
\partial_{t} \boldsymbol{B}_{2}(s, t)=\left[\boldsymbol{Q}, \boldsymbol{\lambda}_{2}(s, t)\right] .
$$

Next, for

$$
\boldsymbol{\Sigma}_{3}(s, t)=\boldsymbol{B}_{3}(s, t) P_{3}-\boldsymbol{B}_{2}(s, t)\left(\Delta(s, t) \pi_{1} \boldsymbol{\Sigma}_{2}(s, t) \wedge P_{0} \pi_{1}\right),
$$

we can find that

$$
\pi_{1} \partial_{t} \boldsymbol{\Sigma}_{3}(s, t)=\left[\boldsymbol{Q}, \pi_{1} \boldsymbol{\rho}_{3}(s, t)\right]
$$

with

$$
\begin{aligned}
\pi_{1} \boldsymbol{\rho}_{3}(s, t)=\pi_{1} \boldsymbol{\lambda}_{3}(s, t) P_{3}-\pi_{1} \boldsymbol{\lambda}_{2}(s, t)\left(\Delta(s, t) \pi_{1} \boldsymbol{\Sigma}_{2}(s, t) \wedge P_{0} \pi_{1}\right) \\
\quad-\pi_{1} \boldsymbol{B}_{2}(s, t)\left(\left[\Delta(s, t) \pi_{1} \boldsymbol{\rho}_{2}(s, t)+Q^{+} \Xi \pi_{1}^{1} \boldsymbol{\Sigma}_{2}(s, t)\right] \wedge P_{0} \pi_{1}\right),
\end{aligned}
$$

using (2.33a) with $n=1$,

$$
\begin{aligned}
\partial_{t} \boldsymbol{B}_{3}(s, t)= & {\left[\boldsymbol{Q}, \boldsymbol{\lambda}_{3}(s, t)\right] } \\
& +\boldsymbol{B}_{2}(s, t)\left(\pi(s) \pi_{1} \boldsymbol{\lambda}_{2}(s, t) \wedge \mathbb{I}_{1}\right)-\boldsymbol{\lambda}_{2}(s, t)\left(\pi(s) \pi_{1} \boldsymbol{B}_{2}(s, t) \wedge \mathbb{I}_{1}\right),
\end{aligned}
$$

and

$$
\partial_{t} \Delta(s, t)=-\left[Q, Q^{+} \Xi \pi^{1}\right]-\Xi P_{0} \pi^{1}
$$


It was also used the off-shell-ness of the internal state. Next, suppose that

$$
\begin{aligned}
\pi_{1} \partial_{t} \boldsymbol{\Sigma}_{l+2}(s, t)= & {\left[\boldsymbol{Q}, \pi_{1} \boldsymbol{\rho}_{l+2}(s, t)\right], } \\
\pi_{1} \boldsymbol{\rho}_{l+2}(s, t)= & \sum_{m=0}^{l} \pi_{1} \boldsymbol{\lambda}_{m+2}(s, t)\left(D_{m+2}(s, t)\right) \pi_{l+2} \\
& -\sum_{m=0}^{l-1} \pi_{1} \boldsymbol{B}_{m+2}(s, t)\left(D_{m+1}(s, t) \wedge \pi_{1} E(s, t)\right) \pi_{l+2},
\end{aligned}
$$

with

$$
E(s, t)=\Delta(s, t) \boldsymbol{\rho}(s, t)+Q^{+} \Xi \pi^{1} \boldsymbol{\Sigma}(s, t),
$$

for $0 \leq l \leq n-1 .^{14}$ Then, we can find that

$$
\pi_{1} \partial_{t} \boldsymbol{\Sigma}_{n+2}(s, t)=\left[\boldsymbol{Q}, \pi_{1} \boldsymbol{\rho}_{n+2}(s, t)\right],
$$

with $\boldsymbol{\rho}_{n+2}(s, t)$ obtained by setting $l=n$ in eq. (D.9). Hence, from the principle of mathematical induction we conclude that $\partial_{t} \Sigma(s, t)=[\boldsymbol{Q}, \boldsymbol{\rho}(s, t)]$ with $\boldsymbol{\rho}(s, t)$ recursively determined by the equation

$$
\begin{aligned}
\pi_{1} \boldsymbol{\rho}(s, t)= & \sum_{m=0}^{\infty} \pi_{1} \boldsymbol{\lambda}_{m+2}(s, t)\left(D_{m+2}(s, t)\right) P_{n+2} \pi_{n+2} \\
& -\sum_{m=0}^{\infty} \pi_{1} \boldsymbol{B}_{m+2}(s, t)\left(D_{m+1}(s, t) \wedge \pi_{1} E(s, t)\right) P_{n+2} \pi_{n+2} .
\end{aligned}
$$

Similarly, for $\boldsymbol{\Sigma}_{2}(s, t)$ and $\boldsymbol{\Sigma}_{3}(s, t)$, we have

$$
\pi_{1} \partial_{s} \boldsymbol{\Sigma}_{2}(s, t)=\left[\boldsymbol{\eta}, \pi_{1} \boldsymbol{\rho}_{2}(s, t)\right], \quad \pi_{1} \partial_{s} \boldsymbol{\Sigma}_{3}(s, t)=\left[\boldsymbol{\eta}, \pi_{1} \boldsymbol{\rho}_{3}(s, t)\right] .
$$

If we assume that

$$
\pi_{1} \partial_{s} \boldsymbol{\Sigma}_{l+2}(s, t)=\left[\boldsymbol{\eta}, \pi_{1} \boldsymbol{\rho}_{l+2}(s, t)\right], \quad \text { for } \quad 0 \leq l \leq n-1,
$$

then, we can show that

$$
\pi_{1} \partial_{s} \boldsymbol{\Sigma}_{n+2}(s, t)=\left[\boldsymbol{\eta}, \pi_{1} \boldsymbol{\rho}_{n+2}(s, t)\right] .
$$

Hence, it is proven from the principle of mathematical induction that $\pi_{1} \partial_{s} \boldsymbol{\Sigma}(s, t)=$ $\left[\boldsymbol{\eta}, \pi_{1} \boldsymbol{\rho}(s, t)\right]$.

\section{E Relation to the Erler-Okawa-Takezaki open superstring field theory}

When starting from the cubic theory, $\boldsymbol{M}_{B}^{(0)}(s)=\left.\boldsymbol{m}_{2}\right|^{0}+\left.s \boldsymbol{m}_{2}\right|^{2}$, it is easy to see that the generating functions $\boldsymbol{A}(s, t)$ and $\boldsymbol{\mu}(s, t)$ of the open superstring product and gauge product have the restricted form

$$
\begin{aligned}
\boldsymbol{A}(s, t) & =\boldsymbol{A}(t)+s \mathcal{A}(t) \\
& =\left.\boldsymbol{A}\right|^{0}(t)+\left.\boldsymbol{A}\right|^{2}(t)+\left.s \mathcal{A}\right|^{0}(t), \\
\boldsymbol{\mu}(s, t) & =\left.\boldsymbol{\mu}\right|^{0}(t),
\end{aligned}
$$

\footnotetext{
${ }^{14}$ Note that the right hand side of (D.9) includes only $\boldsymbol{\Sigma}_{k+2}(s, t)$ and $\boldsymbol{\rho}_{k+2}(s, t)$ with $0 \leq k<l$.
} 
where

$$
\begin{aligned}
& \left.\boldsymbol{A}\right|^{0}(t)=\left.\sum_{n=0}^{\infty} t^{n+1} \boldsymbol{A}_{n+2}^{(n+1)}\right|^{0},\left.\quad \boldsymbol{A}\right|^{2}(t)=\left.\sum_{n=0}^{\infty} t^{n} \boldsymbol{A}_{n+2}^{(n)}\right|^{2} \\
& \left.\mathcal{A}\right|^{0}(t)=\left.\sum_{n=0}^{\infty} t^{n} \mathcal{A}_{n+2}^{(n)}\right|^{0} \\
& \left.\boldsymbol{\mu}\right|^{0}(t)=\left.\sum_{n=0}^{\infty} t^{n} \boldsymbol{\mu}_{n+2}^{(n+1)}\right|^{0} .
\end{aligned}
$$

Then, $[\boldsymbol{A}(s, t), \boldsymbol{\mu}(s, t)]^{2} \equiv 0$ since the gauge product $\boldsymbol{\mu}(s, t)$ is only nonvanishing in the NS sector. The differential equations (4.11) are now simplified and decomposed to the four equations

$$
\begin{aligned}
\left.\partial_{t} \boldsymbol{A}\right|^{0}(t) & =\left[\boldsymbol{Q},\left.\boldsymbol{\mu}\right|^{0}(t)\right]+\left[\left.\boldsymbol{A}\right|^{0}(t),\left.\boldsymbol{\mu}\right|^{0}(t)\right], \\
\left.\partial_{t} \boldsymbol{A}\right|^{2}(t) & =\left[\left.\boldsymbol{A}\right|^{2}(t),\left.\boldsymbol{\mu}\right|^{0}(t)\right], \\
\left.\partial_{t} \mathcal{A}\right|^{0}(t) & =\left[\left.\mathcal{A}\right|^{0}(t),\left.\boldsymbol{\mu}\right|^{0}(t)\right], \\
{\left[\boldsymbol{\eta},\left.\boldsymbol{\mu}\right|^{0}(t)\right] } & =\left.\mathcal{A}\right|^{0}(t),
\end{aligned}
$$

satisfying the initial conditions $\boldsymbol{A}(0)=\left.\boldsymbol{m}_{2}\right|^{2}$ and $\boldsymbol{A}(0)=\left.\boldsymbol{m}_{2}\right|^{0}$. These equations are slight modification of those proposed in ref. [17]. In the previous method, almost the same equations that respect the Ramond number, instead of the cyclic Ramond number, directly provide (the generating function of) the products with $A_{\infty}$ structure. In our method, on the other hand, eqs. (E.5) provide an intermediate products, which have to be transformed to the final form by the cohomomorphism. It can be seen, however, that the final products are the same.

In the present construction, we have an intermediate cyclic $A_{\infty}$ structure $\boldsymbol{Q}-\boldsymbol{\eta}+\boldsymbol{A}(t)$ which split into two (anti-)commutative $A_{\infty}$ structures

$$
\begin{aligned}
& \boldsymbol{D}(t)=\boldsymbol{Q}+\left.\boldsymbol{A}\right|_{0} ^{0}(t)+\left.\boldsymbol{A}\right|_{2} ^{2}(t), \\
& \boldsymbol{C}(t)=\boldsymbol{\eta}-\left.\boldsymbol{A}\right|_{0} ^{2}(t) .
\end{aligned}
$$

Using the fact that $\boldsymbol{A}(t)$ satisfies the differential equations (E.5), they can be rewritten as

$$
\begin{aligned}
& \boldsymbol{D}(t)=\hat{\boldsymbol{g}}(t)^{-1}\left(\boldsymbol{Q}+\left.\boldsymbol{m}_{2}\right|_{2}\right) \hat{\boldsymbol{g}}(t), \\
& \boldsymbol{C}(t)=\hat{\boldsymbol{g}}(t)^{-1}\left(\boldsymbol{\eta}-\left.\boldsymbol{m}_{2}\right|_{0}\right) \hat{\boldsymbol{g}}(t),
\end{aligned}
$$

where the cohomomorphism $\hat{\boldsymbol{g}}(t)$ is given by the path-ordered exponential of $\left.\boldsymbol{\mu}\right|^{0}(t)$ :

$$
\hat{\boldsymbol{g}}(t)=\overrightarrow{\mathcal{P}} \exp \left[\left.\int_{0}^{t} d t^{\prime} \boldsymbol{\mu}\right|^{0}\left(t^{\prime}\right)\right] .
$$

A relation

$$
\hat{\boldsymbol{g}}(t) \boldsymbol{\eta} \hat{\boldsymbol{g}}(t)^{-1}=\boldsymbol{\eta}-\left.\boldsymbol{m}_{2}\right|^{0},
$$

follows from the eq. (E.5d) are also used. The cohomomorphism $\pi_{1} \hat{\boldsymbol{F}}(t)^{-1}=\pi_{1} \mathbb{I}-\Xi \pi_{1}^{1} \boldsymbol{A}(t)$ transforming $\boldsymbol{D}(t)$ to the final $A_{\infty}$ structure $\boldsymbol{M}(t)$, is similarly written as

$$
\hat{\boldsymbol{F}}(t)^{-1}=\hat{\boldsymbol{g}}(t)^{-1}\left(\hat{\boldsymbol{F}}^{-1}\right) \hat{\boldsymbol{g}}(t), \quad \pi_{1} \hat{\boldsymbol{F}}^{-1}=\pi_{1} \mathbb{I}-\left.\Xi \pi_{1}^{1} \boldsymbol{m}_{2}\right|_{0} ^{2},
$$


and thus, we find that

$$
\begin{aligned}
\boldsymbol{M}(t) & =\hat{\boldsymbol{F}}(t)^{-1} \boldsymbol{D}(t) \hat{\boldsymbol{F}}(t)=(\hat{\boldsymbol{F}} \hat{\boldsymbol{g}}(t))^{-1}\left(\boldsymbol{Q}+\left.\boldsymbol{m}_{2}\right|_{2}\right) \hat{\boldsymbol{F}} \hat{\boldsymbol{g}}(t) \\
\boldsymbol{\eta} & =\hat{\boldsymbol{F}}(t)^{-1} \boldsymbol{C}(t) \hat{\boldsymbol{F}}(t)=(\hat{\boldsymbol{F}} \hat{\boldsymbol{g}}(t))^{-1}\left(\boldsymbol{\eta}-\left.\boldsymbol{m}_{2}\right|_{0}\right) \hat{\boldsymbol{F}} \hat{\boldsymbol{g}}(t)
\end{aligned}
$$

It was proven in ref. [23] that the $A_{\infty}$ structure $\boldsymbol{M}=\boldsymbol{M}(1)$ agrees with that given in ref. [17].

\section{F Cyclicity of generalized $A_{\infty}$ structure}

In this appendix, we show that the $A_{\infty}$ algebra (4.15) is cyclic with respect to $\Omega$. First, we note that $\boldsymbol{A}$ is cyclic with respect to $\omega_{l}$ by construction [9]:

$$
\left\langle\omega_{l}\right|\left(\pi_{1} \boldsymbol{A} \otimes \pi_{1}+\pi_{1} \otimes \pi_{1} \boldsymbol{A}\right)=0 .
$$

Then, we can show that $\boldsymbol{a}$ is also cyclic with respect to $\omega_{l}$ as

$$
\begin{aligned}
\left\langle\omega_{l}\right|\left(\pi_{1} \boldsymbol{a} \otimes \pi_{1}+\pi_{1} \otimes \pi_{1} \boldsymbol{a}\right)= & \left\langle\omega_{l}\right|\left(\pi_{1} \boldsymbol{A} \hat{\boldsymbol{F}} \otimes \pi_{1}+\pi_{1} \otimes \pi_{1} \boldsymbol{A} \hat{\boldsymbol{F}}\right) \\
= & \left\langle\omega_{l}\right|\left(\pi_{1} \boldsymbol{A} \hat{\boldsymbol{F}} \otimes\left(\pi_{1} \hat{\boldsymbol{F}}-\Xi \pi_{1}^{1} \boldsymbol{A} \hat{\boldsymbol{F}}\right)+\left(\pi_{1} \hat{\boldsymbol{F}}-\Xi \pi_{1}^{1} \boldsymbol{A} \hat{\boldsymbol{F}}\right) \otimes \pi_{1} \boldsymbol{A} \hat{\boldsymbol{F}}\right) \\
= & \left\langle\omega_{l}\right|\left(\left(\pi_{1} \boldsymbol{A} \otimes \pi_{1}+\pi_{1} \otimes \pi_{1} \boldsymbol{A}\right)(\hat{\boldsymbol{F}} \otimes \hat{\boldsymbol{F}})\right. \\
& \left.\quad+(\mathbb{I} \otimes \Xi-\Xi \otimes \mathbb{I})\left(\pi_{1} \boldsymbol{A} \hat{\boldsymbol{F}} \otimes \pi_{1} \boldsymbol{A} \hat{\boldsymbol{F}}\right)\right) \\
= & 0,
\end{aligned}
$$

where we used the relation

$$
\pi_{1} \mathbb{I}=\pi_{1} \hat{\boldsymbol{F}}-\Xi \pi_{1}^{1} \boldsymbol{A} \hat{\boldsymbol{F}},
$$

following from the definition of $\hat{\boldsymbol{F}}^{-1}$ (4.16), and the fact that $\Xi$ is a BPZ even:

$$
\left\langle\omega_{l}\right|(\mathbb{I} \otimes \Xi-\Xi \otimes \mathbb{I})=0 .
$$

If we note that $[\boldsymbol{\eta}, \boldsymbol{a}]=0$ it is easy to see that $\boldsymbol{a}$ is also cyclic with respect to $\omega_{s}$ :

$$
\begin{aligned}
\left\langle\omega_{s}\right|\left(\pi_{1} \boldsymbol{a} \otimes \mathbb{I}+\mathbb{I} \otimes \pi_{1} \boldsymbol{a}\right) & =\left\langle\omega_{l}\right|\left(\xi_{0} \otimes \mathbb{I}\right)\left(\pi_{1} \boldsymbol{a} \otimes \mathbb{I}+\mathbb{I} \otimes \pi_{1} \boldsymbol{a}\right) \\
& =\sum_{n=0}^{\infty}\left\langle\omega_{l}\right|\left(\xi_{0} \otimes \mathbb{I}\right)\left(\pi_{1} \boldsymbol{a} \otimes \mathbb{I}+\mathbb{I} \otimes \pi_{1} \boldsymbol{a}\right) \pi_{n+3}\left(\left[\eta, \xi_{0}\right] \otimes \mathbb{I}_{n+2}\right) \\
& =-\sum_{n=0}^{\infty}\left\langle\omega_{l}\right|\left(\pi_{1} \boldsymbol{a} \otimes \mathbb{I}+\mathbb{I} \otimes \pi_{1} \boldsymbol{a}\right) \pi_{n+3}\left(\xi_{0} \otimes \mathbb{I}_{n+2}\right) \\
& =0
\end{aligned}
$$

on $\mathcal{H}_{s}$. Since the BRST operator satisfies

$$
\langle\Omega|(Q \otimes \mathbb{I}+\mathbb{I} \otimes Q)=0,
$$

on $\mathcal{H}^{\text {res }}$, we find that

$$
\langle\Omega|\left(\pi_{1} \boldsymbol{M} \otimes \mathbb{I}+\mathbb{I} \otimes \pi_{1} \boldsymbol{M}\right)=\left\langle\omega_{s}\right|\left(\pi_{1} \boldsymbol{a} \otimes \mathbb{I}+\mathbb{I} \otimes \pi_{1} \boldsymbol{a}\right)=0,
$$

on $\mathcal{H}^{\text {res }}$. 
Open Access. This article is distributed under the terms of the Creative Commons Attribution License (CC-BY 4.0), which permits any use, distribution and reproduction in any medium, provided the original author(s) and source are credited.

\section{References}

[1] E. Witten, Interacting Field Theory of Open Superstrings, Nucl. Phys. B 276 (1986) 291 [INSPIRE].

[2] C.R. Preitschopf, C.B. Thorn and S.A. Yost, Superstring Field Theory, Nucl. Phys. B 337 (1990) 363 [INSPIRE].

[3] I.Y. Arefeva, P.B. Medvedev and A.P. Zubarev, New Representation for String Field Solves the Consistency Problem for Open Superstring Field Theory, Nucl. Phys. B 341 (1990) 464 [INSPIRE].

[4] N. Berkovits, SuperPoincaré invariant superstring field theory, Nucl. Phys. B 450 (1995) 90 [Erratum ibid. 459 (1996) 439] [hep-th/9503099] [INSPIRE].

[5] Y. Okawa and B. Zwiebach, Heterotic string field theory, JHEP 07 (2004) 042 [hep-th/0406212] [INSPIRE].

[6] N. Berkovits, Y. Okawa and B. Zwiebach, WZW-like action for heterotic string field theory, JHEP 11 (2004) 038 [hep-th/0409018] [INSPIRE].

[7] B. Jurčo and K. Muenster, Type II Superstring Field Theory: Geometric Approach and Operadic Description, JHEP 04 (2013) 126 [arXiv:1303.2323] [INSPIRE].

[8] Y. Iimori, T. Noumi, Y. Okawa and S. Torii, From the Berkovits formulation to the Witten formulation in open superstring field theory, JHEP 03 (2014) 044 [arXiv:1312.1677] [INSPIRE].

[9] T. Erler, S. Konopka and I. Sachs, Resolving Witten's superstring field theory, JHEP 04 (2014) 150 [arXiv: 1312.2948] [INSPIRE].

[10] T. Erler, S. Konopka and I. Sachs, NS-NS Sector of Closed Superstring Field Theory, JHEP 08 (2014) 158 [arXiv: 1403.0940] [inSPIRE].

[11] H. Matsunaga, Nonlinear gauge invariance and WZW-like action for NS-NS superstring field theory, JHEP 09 (2015) 011 [arXiv:1407.8485] [INSPIRE].

[12] A. Sen, Gauge Invariant 1PI Effective Superstring Field Theory: Inclusion of the Ramond Sector, JHEP 08 (2015) 025 [arXiv:1501.00988] [INSPIRE].

[13] T. Erler, Y. Okawa and T. Takezaki, $A_{\infty}$ structure from the Berkovits formulation of open superstring field theory, arXiv:1505.01659 [INSPIRE].

[14] T. Erler, S. Konopka and I. Sachs, Ramond Equations of Motion in Superstring Field Theory, JHEP 11 (2015) 199 [arXiv:1506.05774] [INSPIRE].

[15] A. Sen, BV Master Action for Heterotic and Type II String Field Theories, JHEP 02 (2016) 087 [arXiv: 1508.05387] [INSPIRE].

[16] H. Kunitomo and Y. Okawa, Complete action for open superstring field theory, Prog. Theor. Exp. Phys. 2016 (2016) 023B01 [arXiv:1508.00366] [INSPIRE].

[17] T. Erler, Y. Okawa and T. Takezaki, Complete Action for Open Superstring Field Theory with Cyclic $A_{\infty}$ Structure, JHEP 08 (2016) 012 [arXiv: 1602.02582] [INSPIRE]. 
[18] S. Konopka and I. Sachs, Open Superstring Field Theory on the Restricted Hilbert Space, JHEP 04 (2016) 164 [arXiv:1602.02583] [INSPIRE].

[19] K. Goto and H. Kunitomo, Construction of action for heterotic string field theory including the Ramond sector, JHEP 12 (2016) 157 [arXiv:1606.07194] [INSPIRE].

[20] T. Erler, Supersymmetry in Open Superstring Field Theory, JHEP 05 (2017) 113 [arXiv: 1610.03251] [INSPIRE].

[21] H. Kunitomo, Space-time supersymmetry in WZW-like open superstring field theory, Prog. Theor. Exp. Phys. 2017 (2017) 043B04 [arXiv: 1612.08508] [INSPIRE].

[22] H. Matsunaga, Notes on the Wess-Zumino-Witten-like structure: $L_{\infty}$ triplet and NS-NS superstring field theory, JHEP 05 (2017) 095 [arXiv: 1612.08827] [INSPIRE].

[23] T. Erler, Superstring Field Theory and the Wess-Zumino-Witten Action, JHEP 10 (2017) 057 [arXiv: 1706 . 02629] [INSPIRE].

[24] H. Kunitomo and T. Sugimoto, Heterotic string field theory with cyclic $L_{\infty}$ structure, Prog. Theor. Exp. Phys. 2019 (2019) 063B02 [Erratum ibid. 2020 (2020) 019201] [arXiv: 1902.02991] [INSPIRE].

[25] H. Kunitomo and T. Sugimoto, Type II superstring field theory with cyclic $L_{\infty}$ structure, Prog. Theor. Exp. Phys. 2020 (2020) 033B06 [arXiv: 1911.04103] [InSPIRE].

[26] D. Friedan, E.J. Martinec and S.H. Shenker, Conformal Invariance, Supersymmetry and String Theory, Nucl. Phys. B 271 (1986) 93 [INSPIRE].

[27] E. Witten, Superstring Perturbation Theory Revisited, arXiv:1209.5461 [INSPIRE].

[28] S.B. Giddings, E.J. Martinec and E. Witten, Modular Invariance in String Field Theory, Phys. Lett. B 176 (1986) 362 [inSPIRE].

[29] M. Saadi and B. Zwiebach, Closed String Field Theory from Polyhedra, Annals Phys. 192 (1989) 213 [INSPIRE].

[30] T. Kugo, H. Kunitomo and K. Suehiro, Nonpolynomial Closed String Field Theory, Phys. Lett. B 226 (1989) 48 [inSPIRE].

[31] B. Zwiebach, Closed string field theory: Quantum action and the Batalin-Vilkovisky master equation, Nucl. Phys. B 390 (1993) 33 [hep-th/9206084] [INSPIRE].

[32] A. Sen and E. Witten, Filling the gaps with PCO's, JHEP 09 (2015) 004 [arXiv: 1504.00609] [INSPIRE].

[33] N. Berkovits and C.T. Echevarria, Four point amplitude from open superstring field theory, Phys. Lett. B 478 (2000) 343 [hep-th/9912120] [INSPIRE].

[34] H. Kunitomo, Y. Okawa, H. Sukeno and T. Takezaki, Fermion scattering amplitudes from gauge-invariant actions for open superstring field theory, arXiv:1612.00777 [INSPIRE].

[35] S. Konopka, The S-matrix of superstring field theory, JHEP 11 (2015) 187 [arXiv: 1507.08250] [INSPIRE].

[36] A. Jevicki and C.-k. Lee, The S Matrix Generating Functional and Effective Action, Phys. Rev. D 37 (1988) 1485 [INSPIRE].

[37] I.Y. Arefeva, L.D. Faddeev and A.A. Slavnov, Generating Functional for the s Matrix in Gauge Theories, Theor. Math. Phys. 21 (1975) 1165 [Teor. Mat. Fiz. 21 (1974) 311] [INSPIRE]. 
[38] T. Erler, Relating Berkovits and $A_{\infty}$ superstring field theories; small Hilbert space perspective, JHEP 10 (2015) 157 [arXiv:1505.02069] [INSPIRE].

[39] H. Erbin, C. Maccaferri, M. Schnabl and J. Vošmera, Classical algebraic structures in string theory effective actions, JHEP 11 (2020) 123 [arXiv:2006.16270] [INSPIRE].

[40] A.S. Arvanitakis, O. Hohm, C. Hull and V. Lekeu, Homotopy Transfer and Effective Field Theory I: Tree-level, arXiv:2007.07942 [INSPIRE].

[41] M. Kohriki, T. Kugo and H. Kunitomo, Gauge Fixing of Modified Cubic Open Superstring Field Theory, Prog. Theor. Phys. 127 (2012) 243 [arXiv:1111.4912] [InSPIRE].

[42] M. Kato and K. Ogawa, Covariant Quantization of String Based on BRS Invariance, Nucl. Phys. B 212 (1983) 443 [INSPIRE].

[43] N. Ohta, Covariant Quantization of Superstrings Based on BRS Invariance, Phys. Rev. D 33 (1986) 1681 [INSPIRE].

[44] M. Ito, T. Morozumi, S. Nojiri and S. Uehara, Covariant Quantization of Neveu-Schwarz-ramond Model, Prog. Theor. Phys. 75 (1986) 934 [InSPIRE].

[45] M. Henneaux, BRST Cohomology of the Fermionic String, Phys. Lett. B 183 (1987) 59 [INSPIRE].

[46] H. Kajiura, Noncommutative homotopy algebras associated with open strings, Rev. Math. Phys. 19 (2007) 1 [math/0306332] [inSPIRE].

[47] M. Kontsevich, Deformation quantization of Poisson manifolds. 1, Lett. Math. Phys. 66 (2003) 157 [q-alg/9709040] [INSPIRE].

[48] M. Markl, Loop homotopy algebras in closed string field theory, Commun. Math. Phys. 221 (2001) 367 [hep-th/9711045] [INSPIRE].

[49] K. Munster and I. Sachs, Quantum Open-Closed Homotopy Algebra and String Field Theory, Commun. Math. Phys. 321 (2013) 769 [arXiv:1109.4101] [InSPIRE].

[50] R. Donagi and E. Witten, Supermoduli Space Is Not Projected, in Proceedings of Symposia in Pure Mathematics 90, American Mathematical Society (2015), p. 19 [arXiv:1304.7798] [INSPIRE].

[51] T. Erler and S. Konopka, Vertical Integration from the Large Hilbert Space, JHEP 12 (2017) 112 [arXiv: 1710.07232] [INSPIRE].

[52] S.F. Moosavian and R. Pius, Hyperbolic Geometry of Superstring Perturbation Theory, arXiv:1703.10563 [INSPIRE].

[53] S.F. Moosavian and R. Pius, Hyperbolic geometry and closed bosonic string field theory. Part I. The string vertices via hyperbolic Riemann surfaces, JHEP 08 (2019) 157 [arXiv: 1706. 07366] [INSPIRE].

[54] S.F. Moosavian and R. Pius, Hyperbolic geometry and closed bosonic string field theory. Part II. The rules for evaluating the quantum BV master action, JHEP 08 (2019) 177 [arXiv: 1708.04977] [INSPIRE].

[55] R. Pius, Quantum Closed Superstring Field Theory and Hyperbolic Geometry I: Construction of String Vertices, arXiv:1808.09441 [INSPIRE].

[56] K. Costello and B. Zwiebach, Hyperbolic String Vertices, arXiv:1909.00033 [InSPIRE]. 
[57] C. de Lacroix, H. Erbin, S.P. Kashyap, A. Sen and M. Verma, Closed Superstring Field Theory and its Applications, Int. J. Mod. Phys. A 32 (2017) 1730021 [arXiv:1703.06410] [INSPIRE].

[58] H. Matsunaga, Light-cone reduction of Witten's open string field theory, JHEP 04 (2019) 143 [arXiv: 1901.08555] [INSPIRE].

[59] J.M. Figueroa-O'Farrill and T. Kimura, The cohomology of BRST complexes, submitted to Commun. Math. Phys., ITP-SB-88-34-REV (1988) [INSPIRE].

[60] J.M. Figueroa-O'Farrill and T. Kimura, The BRST Cohomology of the NSR String: Vanishing and 'No Ghost' Theorems, Commun. Math. Phys. 124 (1989) 105 [INSPIRE].

[61] B.H. Lian and G.J. Zuckerman, BRST Cohomology of the Supervirasoro Algebras, Commun. Math. Phys. 125 (1989) 301 [inSPIRE]. 